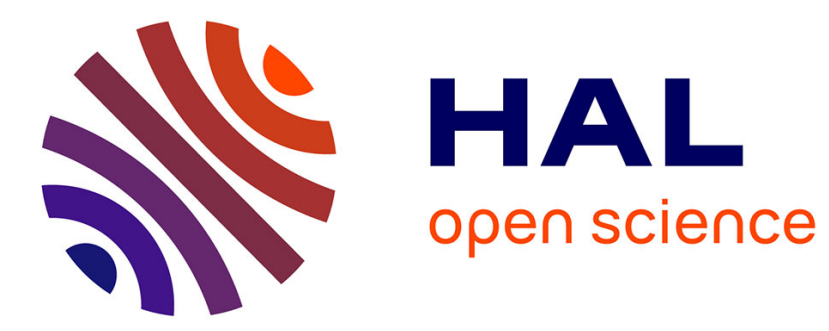

\title{
Random matrix theory for modeling uncertainties in computational mechanics
}

\author{
Christian Soize
}

\section{To cite this version:}

Christian Soize. Random matrix theory for modeling uncertainties in computational mechanics. Computer Methods in Applied Mechanics and Engineering, 2005, 194 (12-16), pp.1333-1366. 10.1016/j.cma.2004.06.038 . hal-00686187

\section{HAL Id: hal-00686187 \\ https://hal.science/hal-00686187}

Submitted on 8 Apr 2012

HAL is a multi-disciplinary open access archive for the deposit and dissemination of scientific research documents, whether they are published or not. The documents may come from teaching and research institutions in France or abroad, or from public or private research centers.
L'archive ouverte pluridisciplinaire HAL, est destinée au dépôt et à la diffusion de documents scientifiques de niveau recherche, publiés ou non, émanant des établissements d'enseignement et de recherche français ou étrangers, des laboratoires publics ou privés. 


\title{
Random matrix theory for modeling uncertainties in computational mechanics
}

\author{
C. Soize \\ Laboratory of Engineering Mechanics, University of Marne-la-Vallée, \\ 5 boulevard Descartes, 77454 Marne-la-Vallée, France, e-mail: soize@univ-mlv.fr
}

\begin{abstract}
This paper deals with data uncertainties and model uncertainties issues in computational mechanics. If data uncertainties can be modeled by parametric probabilistic methods, for a given mean model, a nonparametric probabilistic approach can be used for modeling model uncertainties. The first part is devoted to random matrix theory for which we summarize previous published results and for which two new ensembles of random matrices useful for the nonparametric models are introduced. In a second part, the nonparametric probabilistic approach of random uncertainties is presented for linear dynamical systems and for nonlinear dynamical systems constituted of a linear part with additional localized nonlinearities. In a third part, a new method is proposed for estimating the parameters of the nonparametric approach from experiments. Finally, examples with experimental comparisons are given.
\end{abstract}

Keywords: Computational mechanics, random matrix, random uncertainties, dynamical system, transient, nonlinear

\section{Introduction}

In computational mechanics, random uncertainties in model predictions are due to data uncertainties and to model uncertainties.

Data uncertainties concern the parameters of the mathematical-mechanical model such as the geometrical parameters, the parameters allowing the boundary conditions to be described, the constitutive equations, etc. Therefore, data uncertainties can clearly be taken into account by the 
parametric probabilistic approach whose stochastic finite elements method $[2,9-12,17,26,34,36]$ and other theoretical and numerical methods [11] such as for stochastic eigenvalue problems [21,25,35] constitute very efficient tools in computational mechanics.

Model uncertainties are introduced during the construction of the mathematical-mechanical model: the constructed model cannot exactly represent a complex mechanical system due to the introduction of approximations and simplifications and because the details are unknown, or are not accurately known. Clearly, such model uncertainties are not relevant to the parametric approach because, by definition, the model uncertainties cannot be taken into account by the parameters of the mathematical-mechanical model under consideration (for instance, the thin plate theory does not allow all the types of waves to be modeled). For predictive models of complex systems, a large part of the lack of predictability is due to model uncertainties. For such a complex system: (i) If an additional smaller spatial scale is introduced in the predictive model for reducing model uncertainties, then data uncertainties increase due to the increasing of the number of parameters. (ii) If several spatial scales (or equivalently, a hierarchy of models) are introduced, model uncertainties will always exist for the smaller spatial scale. (iii) For the smaller spatial scale introduced in the predictive model, the model uncertainties cannot be taken into account with the parametric probabilistic approach of data uncertainties. Consequently, in a predictive model of a complex system, there will always have a spatial scale for which model uncertainties will have to be taken into account for increasing the predictability. It should be noted that the objective of this nonparametric probabilistic approach is to increase the predictability of a given and fixed mean model, that is to say, is to increase the predictability without improving the mean model by introducing additional smaller spatial scales or a hierarchy of models. The proposed nonparametric approach also allows the data uncertainties to be modeled, particularly when the number of uncertain parameters becomes large. The nonparametric probabilistic approach of random uncertainties for dynamical sytems was introduced in $[27,29]$. This approach was developed thanks to the introduction of a new ensemble of random matrices that we call the "positive-definite ensemble". This ensemble, which is adapted to the operators of dynamical systems, is constructed and studied in $[28,29]$ and differs from the Gaussian Orthogonal Ensemble (GOE) and from the other known ensembles of the random matrix theory [19] which are not adapted to the mass, damping and stiffness operators of dynamical systems. The bases of the nonparametric probabilistic approach of random uncertainties for linear dynamical systems is presented in $[28,29,31]$ with applications to vibrations and transient elastodynamics. The 
case of uncertain dynamical systems in the medium-frequency range are studied in [32]. Since the nonparametric approach consists in constructing a probability distribution of the random operators of the problem, the case of non homogeneous uncertainties cannot directly be taken into account and dynamic substructuring [4] has to be used. The case of non homogeneous uncertainties for linear dynamical systems with an experimental validation is presented in $[33,3]$. Finally, the case of nonlinear dynamical systems with such a nonparametric model of random uncertainties is developed in [30] and, in [5], a nonparametric-parametric approach is introduced and applied to earthquake engineering analysis of a reactor cooling system under seismic loads.

The first part of this paper is devoted to random matrix ensembles which are used for random uncertainties modeling in computational mechanics. First, for readability of the paper, the results concerning the main ensemble introduced in [28,29] are summarized. In addition, we present two new ensembles of random matrices useful for modeling random uncertainties with the nonparametric approach. For instance, the first one is useful for modeling uncertainties of the mass operator of a dynamical system for which the spatial distribution of the mass is uncertain but for which the total mass is given. The second ensemble, that we call the pseudo-inverse ensemble of random matrices can be used for modeling random uncertainties in the coupling operator between an elastic solid and an acoustic fluid for structural-acoustic systems.

The second part deals with a short overview with the nonparametric probabilistic approach of random uncertainties in linear and nonlinear transient dynamics presented in [28-31]. Nevertheless, we present an additional experimental validation for linear transient dynamics of a non homogeneous structure submitted to a shock.

Finally, in the last part, we propose a new methodology for identification of the dispersion parameter of the nonparametric probabilistic model. Such a method is presented in the context of the experimental modal identification of a linear dynamical system.

\section{Random matrix ensembles for uncertainties modeling in computational mechanics}

The random matrix theory were introduced and developed in mathematical statistics by Wishart and others in the 1930s and was intensively studied by physicists and mathematicians in the context of nuclear physics (Wigner, Dyson, Mehta and others). An excellent synthesis of the random matrix theory can be found in the Mehta book [19]. For physical applications, the most important ensemble of the random matrix theory, is the Gaussian Orthogonal Ensemble (GOE) for which the 
elements are constituted of real symmetric random matrices with statistically independent entries and which are invariant under orthogonal linear transformations. The random matrix theory has been used in other domains that in nuclear physics. In 1989, Weaver [37] show that the higher frequencies of elastodynamic structures constituted of small aluminium blocks have the behavior of the eigenvalues of a matrix belonging to the GOE. These results have clearly been validated for the very high-frequency range in elastodynamics but not at all for the frequency band of interest in structural dynamics, vibration and vibroacoustics systems which are concerned by the low- , medium- and high-frequency ranges.

In this section, we present five ensembles of random matrices which are useful for modeling data and model uncertainties in computational mechanics.

(1) The first ensemble $\mathrm{SG}^{+}$of random matrices, herein called the the normalized positive-definite ensemble, has recently been constructed (see $[28,29]$ ) in the context of the development of a new approach for modeling random uncertainties in dynamical systems with a nonparametric approach. A random matrix belonging to $\mathrm{SG}^{+}$is positive definite almost surely and its mean value is the identity matrix. This ensemble constitutes the main ensemble used for constructing the four other ensembles introduced below. Ensemble $\mathrm{SG}^{+}$differs from the GOE and from the other known ensembles of the random matrix theory. In order to improve the readability of this paper, we will recall the main results concerning this ensemble.

(2) The second ensemble $\mathrm{SE}^{+}$of random matrices, herein called the the positive-definite ensemble, has been constructed in [28,29], simultaneously with $\mathrm{SG}^{+}$. A random matrix belonging to $\mathrm{SE}^{+}$is positive definite almost surely and its mean value is a given positive-definite matrix. For instance, this ensemble is used for constructing probability model of positive operators such as the mass, the damping or the stiffness operators of a dynamical system.

(3) The construction of the third ensemble $\mathrm{SE}^{+0}$ has been introduced in [27] and is similar to the construction of ensemble $\mathrm{SE}^{+}$. A random matrix belonging to this ensemble is semipositive definite almost surely instead of being positive definite almost surely.

(4) The fourth one is the subset $\mathrm{SE}_{\mathrm{lf}}^{+}$of $\mathrm{SE}^{+}$constituted of random matrices in $\mathrm{SE}^{+}$for which a linear form on $\mathrm{SE}^{+}$is given. A particular case is the ensemble $\mathrm{SE}_{\mathrm{tr}}^{+}$for which the trace of the random matrices is given. This is a new ensemble that we construct below. For instance, such an ensemble is useful for modeling uncertainties of the mass operator of dynamical systems for which the spatial distibution of the mass is uncertain but for which the total mass is known. 
(5) The fifth set $\mathrm{SE}_{\mathrm{inv}}$ of random matrices, herein called the the pseudo-inverse ensemble, is a new ensemble that we construct in this paper. This ensemble is constituted of rectangular random matrices having a mean-square pseudo-inverse. For instance, such an ensemble is useful for modeling uncertainties in the coupling operator between an elastic solid and an acoustic fluid for a structural-acoustic system [20].

In this paper, the following algebraic notations are used.

Euclidean space. Let $\mathbf{x}=\left(x_{1}, \ldots, x_{n}\right)$ be a vector in $\mathbb{R}^{n}$. The Euclidean space $\mathbb{R}^{n}$ is equipped with the usual inner product $(\mathbf{x}, \mathbf{y}) \mapsto<\mathbf{x}, \mathbf{y}>=\sum_{j=1}^{n} x_{j} y_{j}$ and the associated norm $\|\mathbf{x}\|=<\mathbf{x}, \mathbf{x}>^{1 / 2}$.

Matrix sets. Let $\mathbb{M}_{n, m}(\mathbb{R})$ be the set of all the $(n \times m)$ real matrices, $\mathbb{M}_{n}(\mathbb{R})=\mathbb{M}_{n, n}(\mathbb{R})$ be the set of all the square $(n \times n)$ real matrices, $\mathbb{M}_{n}^{S}(\mathbb{R})$ be the set of all the $(n \times n)$ real symmetric matrices, $\mathbb{M}_{n}^{+0}(\mathbb{R})$ be the set of all the $(n \times n)$ real symmetric semipositive definite matrices and $\mathbb{M}_{n}^{+}(\mathbb{R})$ be the set of all the $(n \times n)$ real symmetric positive-definite matrices. We then have

$$
\mathbb{M}_{n}^{+}(\mathbb{R}) \subset \mathbb{M}_{n}^{+0}(\mathbb{R}) \subset \mathbb{M}_{n}^{S}(\mathbb{R}) \subset \mathbb{M}_{n}(\mathbb{R})
$$

Norms and usual operators. We denote:

(1) the determinant of matrix $[A] \in \mathbb{M}_{n}(\mathbb{R})$ as $\operatorname{det}[A]$ and its trace $\operatorname{as} \operatorname{tr}[A]=\sum_{j=1}^{n}[A]_{j j}$,

(2) the transpose of $[A] \in \mathbb{M}_{n, m}(\mathbb{R})$ as $[A]^{T} \in \mathbb{M}_{m, n}(\mathbb{R})$,

(3) the operator norm of the matrix $[A] \in \mathbb{M}_{n, m}(\mathbb{R})$ as

$$
\|A\|=\sup _{\|\mathbf{x}\| \leq 1}\|[A] \mathbf{x}\| \quad, \quad \mathbf{x} \in \mathbb{R}^{m}
$$

which is such that $\|[A] \mathbf{x}\| \leq\|A\|\|\mathbf{x}\|, \forall \mathbf{x} \in \mathbb{R}^{m}$,

(4) for $[A]$ and $[B] \in \mathbb{M}_{n, m}(\mathbb{R})$, we denote $\ll[A],[B] \gg=\operatorname{tr}\left\{[A]^{T}[B]\right\}$ and the Frobenius norm (or Hilbert-Schmidt norm) $\|A\|_{F}$ of $[A]$ is such that

$$
\|A\|_{F}^{2}=\ll[A],[A] \gg=\operatorname{tr}\left\{[A]^{T}[A]\right\}=\sum_{j=1}^{n} \sum_{k=1}^{m}[A]_{j k}^{2}
$$

which is such that $\|A\| \leq\|A\|_{F} \leq \sqrt{n}\|A\|$. 


\subsection{Normalized positive-definite ensemble $\mathrm{SG}^{+}$of random matrices}

In this section, we summarize the theory developed in $[28,29]$ concerning the construction of ensemble $\mathrm{SG}^{+}$of random matrices.

\subsubsection{Definition of ensemble $\mathrm{SG}^{+}$}

This ensemble is defined as the random matrices $\left[\mathbf{G}_{n}\right]$, defined on a probability space $(\mathcal{A}, \mathcal{T}, P)$, with values in $\mathbb{M}_{n}^{+}(\mathbb{R})$, whose probability distribution is constructed by using the entropy optimization principle $[24,15]$ for which the constraints (define as the available information) are the following:

(1) Matrix $\left[\mathbf{G}_{n}\right]$ is a symmetric positive-definite real random matrix, that is to say,

$$
\left[\mathbf{G}_{n}\right] \in \mathbb{M}_{n}^{+}(\mathbb{R}) \text { a.s }
$$

(2) Matrix $\left[\mathbf{G}_{n}\right]$ is a second-order random variable,

$$
E\left\{\left\|\left[\mathbf{G}_{n}\right]\right\|^{2}\right\} \leq E\left\{\left\|\left[\mathbf{G}_{n}\right]\right\|_{F}^{2}\right\}<+\infty
$$

(3) The mean value $\left[\underline{G}_{n}\right]$ of random matrix $\left[\mathbf{G}_{n}\right]$ is the $(n \times n)$ identity matrix $\left[I_{n}\right]$,

$$
E\left\{\left[\mathbf{G}_{n}\right]\right\}=\left[\underline{G}_{n}\right]=\left[I_{n}\right] \in \mathbb{M}_{n}^{+}(\mathbb{R})
$$

(4) Random matrix $\left[\mathbf{G}_{n}\right]$ is such that

$$
E\left\{\ln \left(\operatorname{det}\left[\mathbf{G}_{n}\right]\right)\right\}=v \quad \text { with } \quad|v|<+\infty \quad
$$

In Section 2.1.8, we will see that the constraint defined by Eq. (4) yields the following fundamental property for random matrices in ensemble $\mathrm{SG}^{+}$,

$$
E\left\{\left\|\left[\mathbf{G}_{n}\right]^{-1}\right\|_{F}^{2}\right\}<+\infty
$$

It should be noted that Eq. (1) shows that random matrix $\left[\mathbf{G}_{n}\right]$ is invertible almost surely, but since the almost sure convergence does not yield the mean-square convergence, then an additional condition has to be introduced to obtain the property defined by Eq. (5). This is the role plays by Eq. (4). In addition, since for $\theta \in \mathcal{A},\left\|\left[\mathbf{G}_{n}(\theta)\right]^{-1}\right\| \leq\left\|\left[\mathbf{G}_{n}(\theta)\right]^{-1}\right\|_{F}$, then Eq. (5) yields

$$
E\left\{\left\|\left[\mathbf{G}_{n}\right]^{-1}\right\|^{2}\right\}<+\infty
$$




\subsubsection{Dispersion parameter of a random matrix in ensemble $\mathrm{SG}^{+}$}

Let $\delta>0$ be the real parameter defined by

$$
\delta=\left\{\frac{E\left\{\left\|\left[\mathbf{G}_{n}\right]-\left[\underline{G}_{n}\right]\right\|_{F}^{2}\right\}}{\left\|\left[\underline{G}_{n}\right]\right\|_{F}^{2}}\right\}^{1 / 2}=\left\{\frac{1}{n} E\left\{\left\|\left[\mathbf{G}_{n}\right]-\left[I_{n}\right]\right\|_{F}^{2}\right\}\right\}^{1 / 2},
$$

that allows the dispersion of the probability model of random matrix $\left[\mathbf{G}_{n}\right]$ to be fixed. In Ref. [29], it is proved that the dispersion of the probability model is fixed by giving parameter $\delta$ which has to be independent of $n$ and which has to be such that

$$
0<\delta<\sqrt{(n+1)(n+5)^{-1}}
$$

If Eq. (8) does not hold, then Eq. (5) does not hold. Then the constraint defined by Eq. (8) is important for the model.

\subsubsection{Probability distribution of a random matrix in ensemble $\mathrm{SG}^{+}$}

The probability distribution $P_{\left[\mathbf{G}_{n}\right]}$ of random matrix $\left[\mathbf{G}_{n}\right]$ is defined by a probability density function $\left[G_{n}\right] \mapsto p_{\left[\mathbf{G}_{n}\right]}\left(\left[G_{n}\right]\right)$ from $\mathbb{M}_{n}^{+}(\mathbb{R})$ into $\mathbb{R}^{+}=[0,+\infty[$, with respect to the measure (volume element) $\widetilde{d} G_{n}$ on the set $M_{n}^{S}(\mathbb{R})$ such that (see [28] for the construction of this measure),

$$
\widetilde{d} G_{n}=2^{n(n-1) / 4} \Pi_{1 \leq i \leq j \leq n} d\left[G_{n}\right]_{i j}
$$

We then have

$$
P_{\left[\mathbf{G}_{n}\right]}=p_{\left[\mathbf{G}_{n}\right]}\left(\left[G_{n}\right]\right) \widetilde{d} G_{n}
$$

with the normalization condition

$$
\int_{\mathbb{M}_{n}^{+}(\mathbb{R})} p_{\left[\mathbf{G}_{n}\right]}\left(\left[G_{n}\right]\right) \widetilde{d} G_{n}=1
$$

Probability density function $p_{\left[\mathbf{G}_{n}\right]}\left(\left[G_{n}\right]\right)$ is then written $([28,29])$ as

$$
p_{\left[\mathbf{G}_{n}\right]}\left(\left[G_{n}\right]\right)=\mathbb{1}_{\mathbb{M}_{n}^{+}(\mathbb{R})}\left(\left[G_{n}\right]\right) \times C_{\mathbf{G}_{n}} \times\left(\operatorname{det}\left[G_{n}\right]\right)^{(n+1) \frac{\left(1-\delta^{2}\right)}{2 \delta^{2}}} \times \exp \left\{-\frac{(n+1)}{2 \delta^{2}} \operatorname{tr}\left[G_{n}\right]\right\},
$$

in which $\mathbb{1}_{\mathbb{M}_{n}^{+}(\mathbb{R})}\left(\left[G_{n}\right]\right)$ is equal to 1 if $\left[G_{n}\right] \in \mathbb{M}_{n}^{+}(\mathbb{R})$ and is equal to zero if $\left[G_{n}\right] \notin \mathbb{M}_{n}^{+}(\mathbb{R})$ and where positive constant $C_{\mathbf{G}_{n}}$ is such that

$$
C_{\mathbf{G}_{n}}=\frac{(2 \pi)^{-n(n-1) / 4}\left(\frac{n+1}{2 \delta^{2}}\right)^{n(n+1)\left(2 \delta^{2}\right)^{-1}}}{\left\{\Pi_{j=1}^{n} \Gamma\left(\frac{n+1}{2 \delta^{2}}+\frac{1-j}{2}\right)\right\}},
$$

where $\Gamma(z)$ is the gamma function defined for $z>0$ by $\Gamma(z)=\int_{0}^{+\infty} t^{z-1} e^{-t} d t$. Equation (12) shows that $\left\{\left[G_{n}\right]_{j k}, 1 \leq j \leq k \leq n\right\}$ are dependent random variables. 


\subsubsection{Characteristic function of a random matrix in ensemble $\mathrm{SG}^{+}$}

For all $\left[\Theta_{n}\right]$ in $\mathbb{M}_{n}^{S}(\mathbb{R})$, the characteristic function of random matrix $\left[\mathbf{G}_{n}\right]$ with values in $\mathbb{M}_{n}^{+}(\mathbb{R}) \subset$ $\mathbb{M}_{n}^{S}(\mathbb{R})$ is defined by $\Psi_{\left[\mathbf{G}_{n}\right]}\left(\left[\Theta_{n}\right]\right)=E\left\{\exp \left(i \ll\left[\Theta_{n}\right],\left[\mathbf{G}_{n}\right] \gg\right)\right\}$. We then have

$$
\Psi_{\left[\mathbf{G}_{n}\right]}\left(\left[\Theta_{n}\right]\right)=\int_{\mathbb{M}_{n}^{+}(\mathbb{R})} \exp \left(i \operatorname{tr}\left\{\left[\Theta_{n}\right]\left[G_{n}\right]\right\}\right) p_{\left[\mathbf{G}_{n}\right]}\left(\left[G_{n}\right]\right) \widetilde{d} G_{n}
$$

which $([28,29])$ yields

$$
\Psi_{\left[\mathbf{G}_{n}\right]}\left(\left[\Theta_{n}\right]\right)=\left\{\operatorname{det}\left(\left[I_{n}\right]-i \frac{2 \delta^{2}}{n+1}\left[\Theta_{n}\right]\right)\right\}^{-(n+1)\left(2 \delta^{2}\right)^{-1}} .
$$

If $(n+1) / \delta^{2}$ is an integer, then Eq. (15) shows that the probability distribution defined by Eq. (10) with (12) and (13) is a Wishart distribution [1,8]. In general, $(n+1) / \delta^{2}$ is not an integer and consequently, the probability distribution defined by Eq. (10) with (12) and (13) is not a Wishart distribution.

\subsubsection{Second-order moments of a random matrix in ensemble $\mathrm{SG}^{+}$}

Since $\left[\underline{G}_{n}\right]=\left[I_{n}\right]$, the covariance $C_{j k, j^{\prime} k^{\prime}}^{G_{n}}$ of random variables $\left[\mathbf{G}_{n}\right]_{j k}$ and $\left[\mathbf{G}_{n}\right]_{j^{\prime} k^{\prime}}$, defined by

$$
C_{j k, j^{\prime} k^{\prime}}^{G_{n}}=E\left\{\left(\left[\mathbf{G}_{n}\right]_{j k}-\left[I_{n}\right]_{j k}\right)\left(\left[\mathbf{G}_{A_{n}}\right]_{j^{\prime} k^{\prime}}-\left[I_{n}\right]_{j^{\prime} k^{\prime}}\right)\right\}
$$

is written [29] as

$$
C_{j k, j^{\prime} k^{\prime}}^{G_{n}}=\frac{\delta^{2}}{n+1}\left\{\left[I_{n}\right]_{j^{\prime} k}\left[I_{n}\right]_{j k^{\prime}}+\left[I_{n}\right]_{j j^{\prime}}\left[I_{n}\right]_{k k^{\prime}}\right\}
$$

In particular, the variance of random variable $\left[\mathbf{G}_{n}\right]_{j k}$ is such that

$$
V_{j k}^{G_{n}}=\frac{\delta^{2}}{n+1}\left(1+\left[I_{n}\right]_{j k}\right)
$$

\subsubsection{Invariance of ensemble $\mathrm{SG}^{+}$under real orthogonal transformations}

Let $\left[\Phi_{n}\right]$ be any real orthogonal matrix belonging to $\mathbb{M}_{n}(\mathbb{R})$ such that $\left[\Phi_{n}\right]^{T}\left[\Phi_{n}\right]=\left[\Phi_{n}\right]\left[\Phi_{n}\right]^{T}=$ $\left[I_{n}\right]$. Let $\left[\mathbf{G}_{A_{n}}^{\prime}\right]$ be the random matrix with values in $\mathbb{M}_{n}^{+}(\mathbb{R})$ defined by $\left[\mathbf{G}_{n}^{\prime}\right]=\left[\Phi_{n}\right]^{T}\left[\mathbf{G}_{n}\right]\left[\Phi_{n}\right]$. We then have

$$
\left[\mathbf{G}_{n}\right]=\left[\Phi_{n}\right]\left[\mathbf{G}_{n}^{\prime}\right]\left[\Phi_{n}\right]^{T}
$$

The probability density function $p_{\left[\mathbf{G}_{n}^{\prime}\right]}\left(\left[G_{n}^{\prime}\right]\right)$ of random matrix $\left[\mathbf{G}_{n}^{\prime}\right]$, with respect to the volume element $\widetilde{d} G_{n}^{\prime}$ (see Eq. (9)), is such that

$$
p_{\left[\mathbf{G}_{n}^{\prime}\right]}\left(\left[G_{n}^{\prime}\right]\right) \widetilde{d} G_{n}^{\prime}=p_{\left[\mathbf{G}_{n}\right]}\left(\left[G_{n}\right]\right) \widetilde{d} G_{n}
$$


in which $p_{\left[\mathbf{G}_{n}\right]}\left(\left[G_{n}\right]\right)$ is defined by Eq. (12). Let $\left[G_{n}\right]$ and $\left[G_{n}^{\prime}\right]$ be such that $\left[G_{n}\right]=\left[\Phi_{n}\right]\left[G_{n}^{\prime}\right]\left[\Phi_{n}\right]^{T}$. Since $\left[\Phi_{n}\right]$ is a real orthogonal matrix, we deduce that $\widetilde{d} G_{n}=\widetilde{d} G_{n}^{\prime}, \operatorname{det}\left[G_{n}\right]=\operatorname{det}\left[G_{n}^{\prime}\right]$ and $\operatorname{tr}\left[G_{n}\right]=\operatorname{tr}\left[G_{n}^{\prime}\right]$. From Eq. (12), we deduce that

$$
p_{\left[\mathbf{G}_{n}\right]}\left(\left[G_{n}\right]\right) \widetilde{d} G_{n}=p_{\left[\mathbf{G}_{n}\right]}\left(\left[G_{n}^{\prime}\right]\right) \widetilde{d} G_{n}^{\prime}
$$

From Eqs. (20) and (21), we deduce that

$$
p_{\left[\mathbf{G}_{n}^{\prime}\right]}\left(\left[G_{n}^{\prime}\right]\right) \widetilde{d} G_{n}^{\prime}=p_{\left[\mathbf{G}_{n}\right]}\left(\left[G_{n}^{\prime}\right]\right) \widetilde{d} G_{n}^{\prime}
$$

which proves the invariance of random matrix $\left[\mathbf{G}_{n}\right]$ under real orthogonal transformations.

\subsubsection{Algebraic representation of a random matrix in ensemble $\mathrm{SG}^{+}$}

The following algebraic representation of random matrix $\left[\mathbf{G}_{n}\right]$ allows a procedure for the Monte Carlo numerical simulation of random matrix $\left[\mathbf{G}_{n}\right]$ to be defined. With this procedure, the numerical cost induced by the simulation is a constant that depends on dimension $n$ but that is independent of the values of parameter $\delta$. Random matrix $\left[\mathbf{G}_{n}\right]$ can be written as

$$
\left[\mathbf{G}_{n}\right]=\left[\mathbf{L}_{n}\right]^{T}\left[\mathbf{L}_{n}\right]
$$

in which $\left[\mathbf{L}_{n}\right]$ is an upper triangular random matrix with values in $M_{n}(\mathbb{R})$ such that:

(1) random variables $\left\{\left[\mathbf{L}_{n}\right]_{j j^{\prime}}, j \leq j^{\prime}\right\}$ are independent;

(2) for $j<j^{\prime}$, real-valued random variable $\left[\mathbf{L}_{n}\right]_{j j^{\prime}}$ can be written as $\left[\mathbf{L}_{n}\right]_{j j^{\prime}}=\sigma_{n} U_{j j^{\prime}}$ in which $\sigma_{n}=\delta(n+1)^{-1 / 2}$ and where $U_{j j^{\prime}}$ is a real-valued Gaussian random variable with zero mean and variance equal to 1 ;

(3) for $j=j^{\prime}$, positive-valued random variable $\left[\mathbf{L}_{n}\right]_{j j}$ can be written as $\left[\mathbf{L}_{n}\right]_{j j}=\sigma_{n} \sqrt{2 V_{j}}$ in which $\sigma_{n}$ is defined above and where $V_{j}$ is a positive-valued gamma random variable whose probability density function $p_{V_{j}}(v)$ with respect to $d v$ is written as

$$
p_{V_{j}}(v)=\mathbb{1}_{\mathbb{R}^{+}}(v) \frac{1}{\Gamma\left(\frac{n+1}{2 \delta^{2}}+\frac{1-j}{2}\right)} v^{\frac{n+1}{2 \delta^{2}}-\frac{1+j}{2}} e^{-v}
$$


2.1.8. Convergence property of a random matrix in ensemble $\mathrm{SG}^{+}$when dimension goes to infinity It is mathematically proved [29] that $E\left\{\left\|\left[\mathbf{G}_{n}\right]^{-1}\right\|_{F}^{2}\right\}<+\infty$ and therefore that $E\left\{\left\|\left[\mathbf{G}_{n}\right]^{-1}\right\|^{2}\right\}<$ $+\infty$. In addition, the following fundamental property is proved,

$$
\forall n \geq 2 \quad, \quad E\left\{\left\|\left[\mathbf{G}_{n}\right]^{-1}\right\|^{2}\right\} \leq C_{\delta}<+\infty \quad
$$

in which $C_{\delta}$ is a positive finite constant that is independent of $n$ but that depends on $\delta$. Equation (25) means that $n \mapsto E\left\{\left\|\left[\mathbf{G}_{n}\right]^{-1}\right\|^{2}\right\}$ is a bounded function from $\{n \geq 2\}$ into $\mathbb{R}^{+}$. In [29], we have numerically studied the convergence velocity as a function of dispersion parameter $\delta$. Figure 1 shows the graph of function $n \mapsto E\left\{\left\|\left[\mathbf{G}_{A_{n}}\right]^{-1}\right\|^{2}\right\}$ for $\delta_{A}=0.1,0.3$ and 0.5 , constructed by using Eqs. (23)-(24) and the Monte Carlo numerical simulation with 100 realizations. It can be seen that a reasonable convergence is reached for $n \geq 20$ if $\delta=0.1$, for $n \geq 60$ if $\delta=0.3$ and for $n \geq 100$ if $\delta=0.5$.

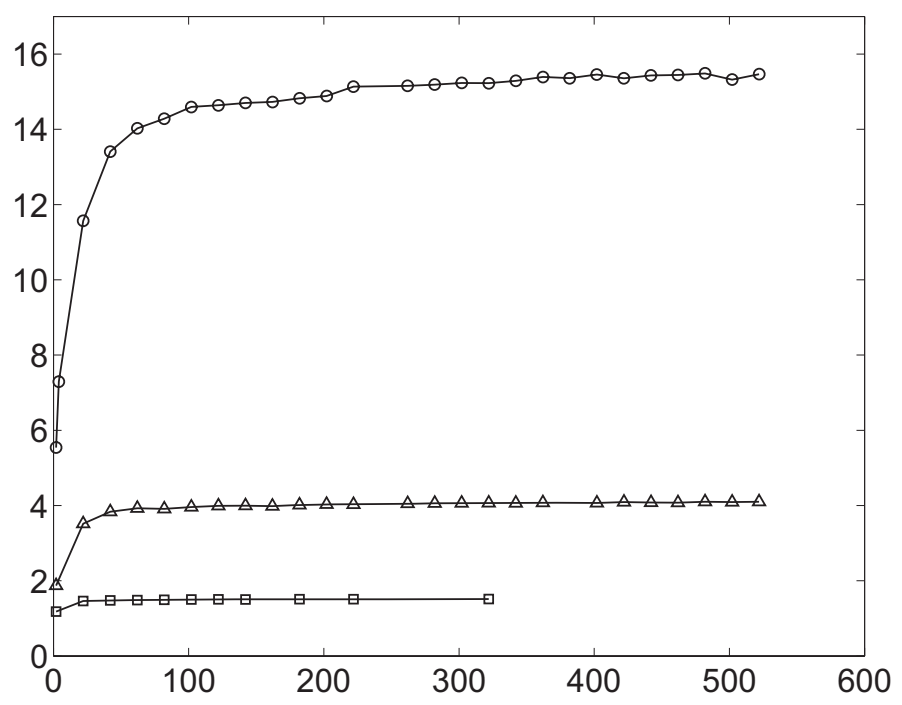

Fig. 1. Graph of function $n \longmapsto E\left\{\left\|\left[\mathbf{G}_{n}\right]^{-1}\right\|^{2}\right\}$ for $\delta=0.1$ (square symbol), $\delta=0.3$ (triangle symbol) and $\delta=0.5$ (circle symbol).

2.1.9. Probability density functions of the random eigenvalues of a random matrix in ensemble $\mathrm{SG}^{+}$

Let $\Lambda_{1}, \ldots, \Lambda_{n}$ be the positive-valued random eigenvalues of random matrix $\left[\mathbf{G}_{n}\right]$. The joint probability density function $p_{\Lambda_{1}, \ldots, \Lambda_{n}}\left(\lambda_{1}, \ldots, \lambda_{n}\right)$ with respect to $d \lambda_{1} \ldots d \lambda_{n}$ of random variables $\Lambda_{1}, \ldots, \Lambda_{n}$ is written [28] as

$$
\begin{aligned}
p_{\Lambda_{1}, \ldots, \Lambda_{n}}\left(\lambda_{1}, \ldots, \lambda_{n}\right) & =\mathbb{1}_{[0,+\infty[}\left(\lambda_{1}\right) \times \ldots \times \mathbb{1}_{[0,+\infty[}\left(\lambda_{n}\right) \times C \times\left(\lambda_{1} \times \ldots \times \lambda_{n}\right)^{(n+1) \frac{\left(1-\delta^{2}\right)}{2 \delta^{2}}} \\
& \times\left\{\Pi_{\alpha<\beta}\left|\lambda_{\beta}-\lambda_{\alpha}\right|\right\} e^{-\frac{(n+1)}{2 \delta^{2}}\left(\lambda_{1}+\ldots+\lambda_{n}\right)}
\end{aligned}
$$

C. Soize - Computer Methods in Applied Mechanics and Engineering (CMAME) (accepted in March 2004) 
in which $C$ is a constant of normalization defined by the equation $\int_{0}^{+\infty} \cdots \int_{0}^{+\infty} p_{\Lambda_{1}, \ldots, \Lambda_{n}}\left(\lambda_{1}, \ldots, \lambda_{n}\right)$ $d \lambda_{1} \ldots d \lambda_{n}=1$. Presently, we are interested in the probability density function of each random eigenvalue for the order statistics. Let $\widetilde{\Lambda}_{1} \leq \widetilde{\Lambda}_{2} \leq \ldots \leq \widetilde{\Lambda}_{n}$ be the order statistics of the random eigenvalues $\Lambda_{1}, \ldots, \Lambda_{n}$. Then, the joint probability density function $p_{\widetilde{\Lambda}_{1}, \ldots, \widetilde{\Lambda}_{n}}\left(\lambda_{1}, \ldots, \lambda_{n}\right)$ with respect to $d \lambda_{1} \ldots d \lambda_{n}$ of random variables $\widetilde{\Lambda}_{1}, \ldots, \widetilde{\Lambda}_{n}$ is written [28] as

$$
p_{\widetilde{\Lambda}_{1}, \ldots, \widetilde{\Lambda}_{n}}\left(\lambda_{1}, \ldots, \lambda_{n}\right)=\frac{\mathbb{1}_{\mathcal{S}_{n}}\left(\lambda_{1}, \ldots, \lambda_{n}\right) p_{\Lambda_{1}, \ldots, \Lambda_{n}}\left(\lambda_{1}, \ldots, \lambda_{n}\right)}{\int_{\mathcal{S}_{n}} p_{\Lambda_{1}, \ldots, \Lambda_{n}}\left(\lambda_{1}, \ldots, \lambda_{n}\right) d \lambda_{1} \ldots d \lambda_{n}}
$$

in which $\mathcal{S}_{n}$ is the simplex defined by

$$
\mathcal{S}_{n}=\left\{\left(\lambda_{1}, \ldots, \lambda_{n}\right) \in \mathbb{R}^{n} ; 0<\lambda_{1}<\ldots<\lambda_{n}<+\infty\right\}
$$

The probability density function $p_{\widetilde{\Lambda}_{j}}\left(\lambda_{j}\right)$ with respect to $d \lambda_{j}$ of random variable $\widetilde{\Lambda}_{j}$ can then be written as

$$
p_{\widetilde{\Lambda}_{j}}\left(\lambda_{j}\right)=\int_{\text {except over } \lambda_{j}} p_{\widetilde{\Lambda}_{1}, \ldots, \widetilde{\Lambda}_{n}}\left(\lambda_{1}, \ldots, \lambda_{n}\right) d \lambda_{1} \ldots d \lambda_{j-1} d \lambda_{j+1} \ldots d \lambda_{n}
$$

An explicit calculation of $p_{\widetilde{\Lambda}_{j}}\left(\lambda_{j}\right)$ cannot be performed. An estimation can be constructed by using the Monte Carlo numerical simulation. Figure 2 shows the graphs of probability density functions $p_{\widetilde{\Lambda}_{j}}$ for $j=1, \ldots n$ with $\delta=0.5, n=30$ and with 10000 realizations in the Monte Carlo numerical simulation. This figure shows that the dispersion of random eigenvalue $\widetilde{\Lambda}_{j}$ increases with its rank $j$.

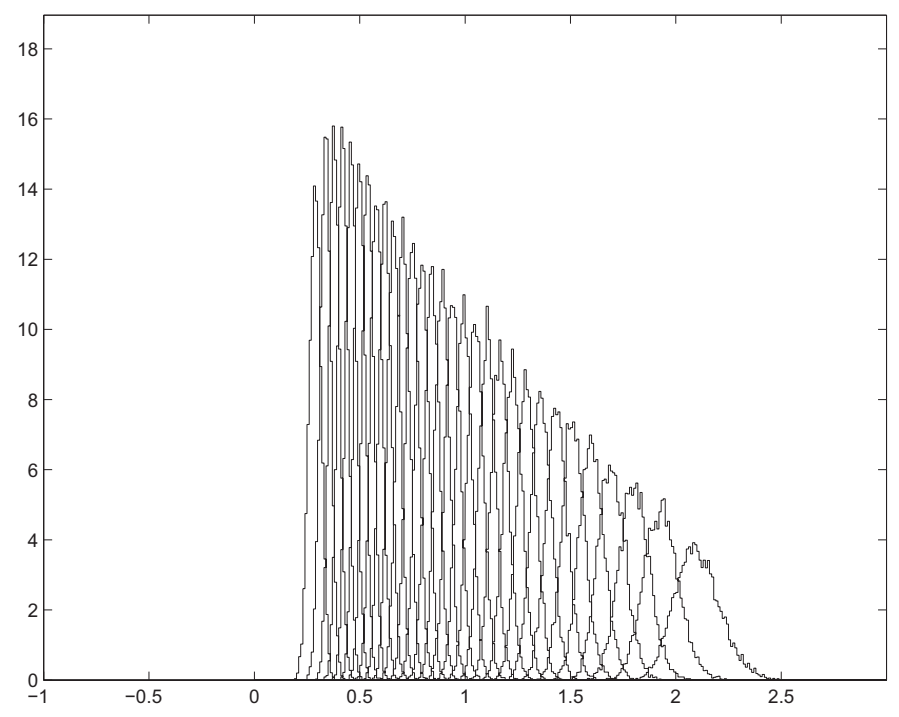

Fig. 2. Graphs of probability density functions $p_{\Lambda_{j}}$ for $j=1, \ldots n$ corresponding to the order statistics of the random eigenvalues of random matrix $\left[\mathbf{G}_{n}\right]$. 


\subsection{Ensemble $\mathrm{SE}^{+}$of random matrices}

\subsubsection{Definition of ensemble $\mathrm{SE}^{+}$}

The ensemble $\mathrm{SE}^{+}$is defined as the set of the random matrices $\left[\mathbf{A}_{n}\right]$, defined on probability space $(\mathcal{A}, \mathcal{T}, P)$, with values in $\mathbb{M}_{n}^{+}(\mathbb{R})$, having similar properties that the properties defined by Eqs. (1), (2) and (4), but for which

$$
E\left\{\left[\mathbf{A}_{n}\right]\right\}=\left[\underline{A}_{n}\right] \in \mathbb{M}_{n}^{+}(\mathbb{R})
$$

in which the mean value of $\left[\mathbf{A}_{n}\right]$ is a given matrix $\left[\underline{A}_{n}\right]$ in $\mathbb{M}_{n}^{+}(\mathbb{R})$. Since matrix $\left[\underline{A}_{n}\right]$ is positive definite, there is an upper triangular matrix $\left[\underline{L}_{A_{n}}\right]$ in $\mathbb{M}_{n}(\mathbb{R})$ such that

$$
\left[\underline{A}_{n}\right]=\left[\underline{L}_{A_{n}}\right]^{T}\left[\underline{L}_{A_{n}}\right],
$$

which corresponds to the Cholesky factorization of matrix $\left[\underline{A}_{n}\right]$. Consequently, ensemble $\mathrm{SE}^{+}$is defined as the set of matrices $\left[\mathbf{A}_{n}\right]$ which are written as

$$
\left[\mathbf{A}_{n}\right]=\left[\underline{L}_{A_{n}}\right]^{T}\left[\mathbf{G}_{n}\right]\left[\underline{L}_{A_{n}}\right]
$$

in which matrix $\left[\mathbf{G}_{n}\right]$ is the random matrix in ensemble $\mathrm{SG}^{+}$.

\subsubsection{Properties of a random matrix in ensemble $\mathrm{SE}^{+}$}

Taking into account Eqs. (1) to (5), it can be deduced that random matrix $\left[\mathbf{A}_{n}\right]$ has the following properties:

(1) Matrix $\left[\mathbf{A}_{n}\right]$ is a symmetric positive-definite real random matrix, that is to say,

$$
\left[\mathbf{A}_{n}\right] \in \mathbb{M}_{n}^{+}(\mathbb{R}) \text { a.s }
$$

(2) Matrix $\left[\mathbf{A}_{n}\right]$ is a second-order random variable,

$$
E\left\{\left\|\left[\mathbf{A}_{n}\right]\right\|_{F}^{2}\right\}<+\infty
$$

(3) The mean value of random matrix $\left[\mathbf{A}_{n}\right]$ is such that

$$
E\left\{\left[\mathbf{A}_{n}\right]\right\}=\left[\underline{A}_{n}\right] \in \mathbb{M}_{n}^{+}(\mathbb{R})
$$

(4) Random matrix $\left[\mathbf{A}_{n}\right]$ is such that

$$
E\left\{\left\|\left[\mathbf{A}_{n}\right]^{-1}\right\|^{2}\right\} \leq E\left\{\left\|\left[\mathbf{A}_{n}\right]^{-1}\right\|_{F}^{2}\right\}<+\infty
$$




\subsubsection{Dispersion parameter of a random matrix in ensemble $\mathrm{SE}^{+}$}

The dispersion is controlled by parameter $\delta$ defined by Eq. (7) and verifying Eq. (8), that we rewrite as $\delta_{A}>0$, and which is such that

$$
\delta_{A}=\left\{\frac{1}{n} E\left\{\left\|\left[\mathbf{G}_{n}\right]-\left[I_{n}\right]\right\|_{F}^{2}\right\}\right\}^{1 / 2} .
$$

Parameter $\delta_{A}$, which has to be independent of $n$ and which has to be chosen such that

$$
0<\delta_{A}<\sqrt{(n+1)(n+5)^{-1}}
$$

allows the dispersion of the probability model of random matrix $\left[\mathbf{A}_{n}\right]$ to be fixed. Finally, the algebraic representation of random matrix $\left[\mathbf{A}_{n}\right]$ is given by Eqs. (32) and (23)-(24).

\subsubsection{Probability model of a set of random matrices in ensemble $\mathrm{SE}^{+}$}

Let us consider $\nu$ random matrices $\left[\mathbf{A}_{n}^{1}\right], \ldots,\left[\mathbf{A}_{n}^{\nu}\right]$ belonging to ensemble $\mathrm{SE}^{+}$. This means that the mean values of the random matrices are known but that no information is available concerning correlation tensor between two any random matrices such as $\left[\mathbf{A}_{j}^{\nu}\right]$ and $\left[\mathbf{A}_{k}^{\nu}\right]$. Then, applying the maximum entropy principle, it can be proved that the probability density function $\left(\left[A_{n}^{1}\right], \ldots,\left[A_{n}^{\nu}\right]\right) \mapsto p_{\left[\mathbf{A}_{n}^{1}\right], \ldots,\left[\mathbf{A}_{n}^{\nu}\right]}\left(\left[A_{n}^{1}\right], \ldots,\left[A_{n}^{\nu}\right]\right)$ from $\mathbb{M}_{n}^{+}(\mathbb{R}) \times \ldots \times \mathbb{M}_{n}^{+}(\mathbb{R})$ into $\mathbb{R}^{+}$with respect to the measure (volume element) $\widetilde{d} A_{n}^{1} \times \ldots \times \widetilde{d} A_{n}^{\nu}$ on $\mathbb{M}_{n}^{S}(\mathbb{R}) \times \ldots \times \mathbb{M}_{n}^{S}(\mathbb{R})$ is written as

$$
p_{\left[\mathbf{A}_{n}^{1}\right], \ldots,\left[\mathbf{A}_{n}^{\nu}\right]}\left(\left[A_{n}^{1}\right], \ldots,\left[A_{n}^{\nu}\right]\right)=p_{\left[\mathbf{A}_{n}^{1}\right]}\left(\left[A_{n}^{1}\right]\right) \times \ldots \times p_{\left[\mathbf{A}_{n}^{\nu}\right]}\left(\left[A_{n}^{\nu}\right]\right)
$$

which means that $\left[\mathbf{A}_{n}^{1}\right], \ldots,\left[\mathbf{A}_{n}^{\nu}\right]$ are independent random matrices.

\subsection{Ensemble $\mathrm{SE}^{+0}$ of random matrices}

The ensemble $\mathrm{SE}^{+0}$ is defined as the set of the second-order random matrices $\left[\mathbf{A}_{m}\right]$, defined on probability space $(\mathcal{A}, \mathcal{T}, P)$, with values in $M_{m}^{+0}(\mathbb{R})$, such that

$$
E\left\{\left[\mathbf{A}_{m}\right]\right\}=\left[\underline{A}_{m}\right] \in \mathbb{M}_{m}^{+0}(\mathbb{R})
$$

in which the mean value of $\left[\mathbf{A}_{m}\right]$ is a given matrix $\left[\underline{A}_{m}\right]$ in $\mathbb{M}_{m}^{+0}(\mathbb{R})$. Since matrix $\left[\underline{A}_{m}\right]$ is semipositive definite, there is a rectangular matrix $\left[\underline{L}_{A_{m}}\right]$ in $\mathbb{M}_{n, m}(\mathbb{R})$ such that

$$
\left[\underline{A}_{m}\right]=\left[\underline{L}_{A_{m}}\right]^{T}\left[\underline{L}_{A_{m}}\right]
$$

C. Soize - Computer Methods in Applied Mechanics and Engineering (CMAME) (accepted in March 2004) 
in which

$$
n=m-\mu_{\text {rig }} \quad,
$$

where $\mu_{\text {rig }} \geq 1$ is the dimension of the null space of $\left[\underline{A}_{n}\right]$. The computation of this factorization will not be explained here but can be deduced from the usual decompositions [13]. The ensemble $\mathrm{SE}^{+0}$ is then defined as the set of matrices $\left[\mathbf{A}_{m}\right]$ which are written as

$$
\left[\mathbf{A}_{m}\right]=\left[\underline{L}_{A_{m}}\right]^{T}\left[\mathbf{G}_{n}\right]\left[\underline{L}_{A_{m}}\right]
$$

in which matrix $\left[\mathbf{G}_{n}\right]$ is the random matrix in ensemble $\mathrm{SG}^{+}$. Clearly, we have

$$
E\left\{\left\|\left[\mathbf{A}_{m}\right]\right\|_{F}^{2}\right\}<+\infty
$$

The dispersion is controlled by parameter $\delta_{A}$ defined by Eq. (37) and verifying Eq. (38).

\subsection{Ensembles $\mathrm{SE}_{\mathrm{lf}}^{+}$and $\mathrm{SE}_{\mathrm{tr}}^{+}$of random matrices}

\subsubsection{Definition of ensembles $\mathrm{SE}_{1 \mathrm{f}}^{+}$and $\mathrm{SE}_{\mathrm{tr}}^{+}$}

Let $\left[B_{n}\right] \mapsto f\left(\left[B_{n}\right]\right)$ be a linear form on $\mathbb{M}_{n}^{+}(\mathbb{R})$ with values in $] 0,+\infty[$. For instance $f$ can be the trace which is such that, for all $\left[B_{n}\right]$ in $M_{n}^{+}(\mathbb{R})$, we have $\operatorname{tr}\left[B_{n}\right]>0$. More generally, linear form $f$ can be written as $f\left(\left[B_{n}\right]\right)=\operatorname{tr}\left\{[\underline{S}]^{T}\left[B_{n}\right][\underline{S}]\right\}$ in which $[\underline{S}] \in \mathbb{M}_{n, \nu}(\mathbb{R})$ with $\nu \geq 1$ and where $[\underline{S}]$ differs from the zero matrix. The ensemble $\mathrm{SE}_{1 \mathrm{f}}^{+}$is defined as follows. Each random matrix $\left[\mathbf{B}_{n}\right]$ belonging to $\mathrm{SE}_{\text {lf }}^{+}$is a second-order random matrix, defined on probability space $(\mathcal{A}, \mathcal{T}, P)$, with values in $M_{n}^{+}(\mathbb{R})$, whose mean value is

$$
E\left\{\left[\mathbf{B}_{n}\right]\right\}=\left[\underline{B}_{n}\right] \in \mathbb{M}_{n}^{+}(\mathbb{R})
$$

and which is written as

$$
\left[\mathbf{B}_{n}\right]=\underline{b}_{n} \frac{1}{f\left(\left[\mathbf{A}_{n}\right]\right)}\left[\mathbf{A}_{n}\right]
$$

in which the random matrix $\left[\mathbf{A}_{n}\right]$ with values in $M_{n}^{+}(\mathbb{R})$ belong to $\mathrm{SE}^{+}$and is written as

$$
\left[\mathbf{A}_{n}\right]=\left[\underline{U}_{n}\right]^{T}\left[\mathbf{G}_{n}\right]\left[\underline{U}_{n}\right]
$$

where $\left[\underline{U}_{n}\right]$ is an invertible matrix in $\mathbb{M}_{n}(\mathbb{R}]$ such that

$$
\left[\underline{B}_{n}\right]=\underline{b}_{n} E\left\{\frac{1}{f\left(\left[\mathbf{A}_{n}\right]\right)}\left[\mathbf{A}_{n}\right]\right\}
$$

C. Soize - Computer Methods in Applied Mechanics and Engineering (CMAME) (accepted in March 2004) 
and where the random matrix $\left[\mathbf{G}_{n}\right]$ belongs to $\mathrm{SG}^{+}$. It should be noted that, if $\left[\underline{U}_{n}\right]$ verifies Eq. (48), then $\mu\left[\underline{U}_{n}\right]$ verifies Eq. (48) for all real $\mu$. In addition, the definition of $\mathrm{SE}_{\mathrm{lf}}^{+}$is consistent if, for any $\left[\underline{B}_{n}\right]$ given in $M_{n}^{+}(\mathbb{R})$, Eq. (48) has at least a solution. If $f\left(\left[B_{n}\right]\right)=\operatorname{tr}\left[B_{n}\right]$, then $\mathrm{SE}_{\mathrm{lf}}^{+}$is denoted by $\mathrm{SE}_{\mathrm{tr}}^{+}$. Since matrix $\left[\underline{B}_{n}\right]$ is positive definite, there is an upper triangular matrix $\left[\underline{L}_{B_{n}}\right]$ in $\mathbb{M}_{n}(\mathbb{R})$ such that

$$
\left[\underline{B}_{n}\right]=\left[\underline{L}_{B_{n}}\right]^{T}\left[\underline{L}_{B_{n}}\right]
$$

which corresponds to the Cholesky factorization of matrix $\left[\underline{B}_{n}\right]$. Consequently, random matrix $\left[\mathbf{B}_{n}\right]$ can be rewritten as

$$
\left[\mathbf{B}_{n}\right]=\left[\underline{L}_{B_{n}}\right]^{T}\left[\mathbf{H}_{n}\right]\left[\underline{L}_{B_{n}}\right]
$$

in which the random matrix $\left[\mathbf{H}_{n}\right]$ with values in $M_{n}^{+}(\mathbb{R})$ is a second-order random variable whose mean value is

$$
E\left\{\left[\mathbf{H}_{n}\right]\right\}=\left[I_{n}\right]
$$

and which is written as

$$
\left[\mathbf{H}_{n}\right]=\underline{b}_{n} \frac{1}{f\left(\left[\mathbf{A}_{n}\right]\right)}\left[\underline{L}_{B_{n}}\right]^{-T}\left[\mathbf{A}_{n}\right]\left[\underline{L}_{B_{n}}\right]^{-1}
$$

where $\left[\mathbf{A}_{n}\right]$ is defined by Eq. (47).

\subsubsection{Properties of a random matrix in ensemble $\mathrm{SE}_{\mathrm{lf}}^{+}$}

It can easily be deduced that second-order random matrix $\left[\mathbf{B}_{n}\right]$ with values in $\mathcal{M}_{n}^{+}(\mathbb{R})$ with $E\left\{\left[\mathbf{B}_{n}\right]\right\}=$ $\left[\underline{B}_{n}\right] \in \mathbb{M}_{n}^{+}(\mathbb{R})$, is such that

$$
\begin{gathered}
f\left(\left[\mathbf{B}_{n}\right]\right)=\underline{b}_{n} \quad \text { a.s }, \\
E\left\{\left\|\left[\mathbf{B}_{n}\right]^{-1}\right\|^{2}\right\}<+\infty .
\end{gathered}
$$

As previously, the dispersion of random matrix $\left[\mathbf{B}_{n}\right]$ is controlled by $\delta_{B}$ such that

$$
\delta_{B}=\left\{\frac{1}{n} E\left\{\left\|\left[\mathbf{G}_{n}\right]-\left[I_{n}\right]\right\|_{F}^{2}\right\}\right\}^{1 / 2},
$$

in which $\delta_{B}$ has to be independent of $n$ and such that $0<\delta_{B}<\sqrt{(n+1)(n+5)^{-1}}$. 


\subsubsection{Algorithm for the construction}

In this subsection, we present an algorithm for constructing matrix $\left[\underline{U}_{n}\right]$. We introduce the invertible matrices $\left[\underline{V}_{n}\right]$ and $\left[\underline{W}_{n}\right]$ in $\mathbb{M}_{n}(\mathbb{R})$ such that

$$
\left[\underline{V}_{n}\right]=\left[\underline{U}_{n}\right]\left[\underline{L}_{B_{n}}\right]^{-1} \quad, \quad\left[\underline{W}_{n}\right]=\left[\underline{V}_{n}\right]\left[\underline{V}_{n}\right]^{T} \quad
$$

Therefore, Eqs. (48) can be rewritten as

$$
\left[\underline{W}_{n}\right]^{-1}=\underline{b}_{n} E\left\{\frac{\left[\mathbf{G}_{n}\right]}{f\left(\left[\mathbf{A}_{n}\right]\right)}\right\}
$$

in which

$$
\left[\mathbf{A}_{n}\right]=\left[\underline{U}_{n}\right]^{T}\left[\mathbf{G}_{n}\right]\left[\underline{U}_{n}\right] \quad, \quad\left[\underline{U}_{n}\right]=\left[\underline{V}_{n}\right]\left[\underline{L}_{B_{n}}\right] .
$$

The construction of a solution $\left[\underline{U}_{n}\right]$ of Eqs. (56)-(57) is performed with the following iterative algorithm.

- Initialization: $\left[\underline{W}_{n}^{(0)}\right]=\left[I_{n}\right]$.

- Calculations for iteration $(j)$ for which matrix $\left[\underline{W}_{n}^{(j)}\right]$ is known.

1. Calculation of $\left[\underline{V}_{n}^{(j)}\right]$ such that $\left[\underline{W}_{n}^{(j)}\right]=\left[\underline{V}_{n}^{(j)}\right]\left[\underline{V}_{n}^{(j)}\right]$.

2. Calculation of $\left[\underline{U}_{n}^{(j)}\right]=\left[\underline{V}_{n}^{(j)}\right]\left[\underline{L}_{B_{n}}\right]$.

3. Calculation of

$$
\begin{aligned}
& {\left[\underline{B}_{n}^{(j)}\right]=\underline{b}_{n} E\left\{\left[\mathbf{A}_{n}^{(j)}\right] / f\left(\left[\mathbf{A}_{n}^{(j)}\right]\right)\right\}} \\
& {\left[\underline{R}_{n}^{(j+1)}\right]=\underline{b}_{n} E\left\{\left[\mathbf{G}_{n}\right] / f\left(\left[\mathbf{A}_{n}^{(j)}\right]\right)\right\}} \\
& \text { with }\left[\mathbf{A}_{n}^{(j)}\right]=\left[\underline{U}_{n}\right]^{T}\left[\mathbf{G}_{n}\right]\left[\underline{U}_{n}^{(j)}\right] .
\end{aligned}
$$

4. Convergence test with $\varepsilon^{(j)}=\left\|\left[\underline{B}_{n}\right]-\left[\underline{B}_{n}^{(j)}\right]\right\| /\left\|\left[\underline{B}_{n}\right]\right\|$ :

$$
\begin{aligned}
& \text { If } \varepsilon^{(j)} \leq \varepsilon_{0} \text { then }\left[\underline{U}_{n}\right]=\left[\underline{U}_{n}^{(j)}\right] \text { and go to } 8 . \\
& \text { If } \varepsilon^{(j)}>\varepsilon_{0} \text { then go to } 5 \text {. }
\end{aligned}
$$

5. Calculation of $\left[\underline{W}_{n}^{(j+1)}\right]=\left[\underline{R}_{n}^{(j+1)}\right]^{-1}$.

6. Loading $\left[\underline{W}_{n}^{(j+1)}\right]$ in $\left[\underline{W}_{n}^{(j)}\right]$.

7. Go to 1 .

8. End 


\subsubsection{Normalized case}

We consider the normalized case defined by $\left[\underline{B}_{n}\right]=\left[I_{n}\right]$ with $n=6$ and $f=$ tr. Then, $\left[\mathbf{B}_{n}\right]=\left[\mathbf{H}_{n}\right]$, we have $\underline{b}_{n}=\operatorname{tr}\left[I_{n}\right]=6$ and $\left\|\left[\underline{B}_{n}\right]\right\|=1$. Three values of the dispersion parameter are considered: $\delta_{B}=0.1,0.3$ and 0.5. The convergence of the iteration algorithm for the construction of matrix $\left[\underline{U}_{n}\right]$ is analyzed in computing $\varepsilon^{(j)}=\left\|\left[\underline{B}_{n}\right]-\left[\underline{B}_{n}^{(j)}\right]\right\| /\left\|\left[\underline{B}_{n}\right]\right\|$ as a function of the iteration number $j$. Figure 3 displays the graphs of functions $j \mapsto \log 10\left(\varepsilon^{(j)}\right)$ for $\delta_{B}=0.1,0.3$ and 0.5 . It can be seen that for the three values of the dispersion parameter, convergence is reached with 7 iterations with a relative error less than $10^{-12}$.

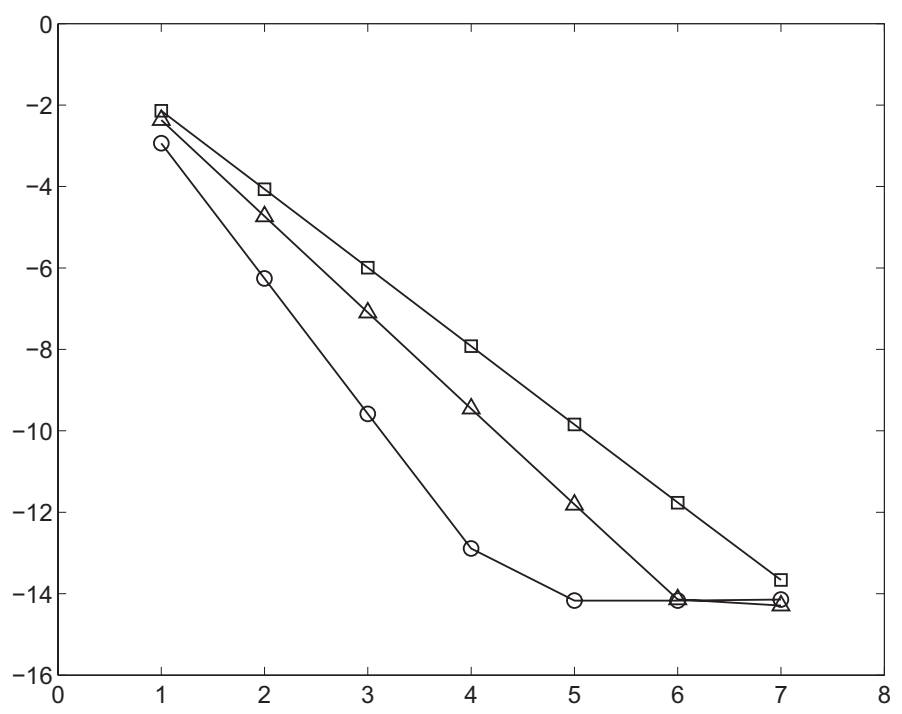

Fig. 3. Convergence of the iteration algorithm. Graphs of functions $j \mapsto \log 10\left(\varepsilon^{(j)}\right)$ with $\varepsilon^{(j)}=\left\|\left[\underline{B}_{n}\right]-\left[\underline{B}_{n}^{(j)}\right]\right\| /\left\|\left[\underline{B}_{n}\right]\right\|$ for $\delta_{B}=0.1$ (circle), $\delta_{B}=0.3$ (triangle), $\delta_{B}=0.5$ (square).

For $\delta_{B}=0.5$, matrix $\left[\underline{U}_{n}\right]$ obtained at convergence by the iteration algorithm is written as

$$
\left[\underline{U}_{n}\right]=\left[\begin{array}{cccccc}
0.9992 & 0 & 0 & 0 & 0 & 0 \\
-0.0023 & 1.0015 & 0 & 0 & 0 & 0 \\
-0.0003 & -0.0012 & 1.0008 & 0 & 0 & 0 \\
-0.0040 & 0.0004 & 0.0020 & 0.9998 & 0 & 0 \\
-0.0013 & -0.0021 & 0.0004 & -0.0024 & 0.9987 & 0 \\
0.0004 & -0.0010 & 0.0008 & 0.0011 & -0.0016 & 0.9999
\end{array}\right]
$$

In this case, $\left[\underline{U}_{n}\right]$ is a lower triangular matrix.

\subsection{Ensemble $\mathrm{SE}_{\mathrm{inv}}$ of random matrices}

\subsubsection{Decomposition of a rectangular matrix}

Let $\left[\underline{A}_{m, n}\right]$ be a rectangular real matrix in $\mathbb{M}_{m, n}(\mathbb{R})$ for which its null space is reduced to $\{0\}$, that is to say such that

$$
\left[\underline{A}_{m, n}\right] \mathbf{x}=0 \quad \Longrightarrow \quad \mathbf{x}=0
$$

C. Soize - Computer Methods in Applied Mechanics and Engineering (CMAME) (accepted in March 2004) 
Then, rectangular matrix $\left[\underline{A}_{m, n}\right]$ can be written as

$$
\left[\underline{A}_{m, n}\right]=\left[\underline{U}_{m, n}\right]\left[\underline{T}_{n}\right],
$$

in which the rectangular matrix $\left[\underline{U}_{m, n}\right]$ and the symmetric square matrix $\left[\underline{T}_{n}\right]$ are such that

$$
\left[\underline{T}_{n}\right] \in \mathbb{M}_{n}^{+}(\mathbb{R}) \quad \text { and } \quad\left[\underline{U}_{m, n}\right] \in \mathbb{M}_{m, n}(\mathbb{R}) \quad \text { such that } \quad\left[\underline{U}_{m, n}\right]^{T}\left[\underline{U}_{m, n}\right]=\left[I_{n}\right]
$$

\subsubsection{Construction of the decomposition of a rectangular matrix}

The construction of the decomposition defined by Eq. (60) can be performed by using the singular value decomposition [13]. Nevertheless, we give below a direct construction which is similar to the construction used to construct the decomposition $\mathbb{U} \mathbb{T}$ of a compact operator $\mathbb{A}[14]$.

For a given rectangular matrix $\left[\underline{A}_{m, n}\right] \in M_{m, n}(\mathbb{R})$ with a null space reduced to $\{0\}$ (see Eq. (59)), let $\left[\underline{B}_{n}\right]$ be the positive-definite square matrix such that

$$
\left[\underline{B}_{n}\right]=\left[\underline{A}_{m, n}\right]^{T}\left[\underline{A}_{m, n}\right] \in \mathbb{M}_{n}^{+}(\mathbb{R})
$$

It should be noted that $\left[\underline{B}_{n}\right]$ is a positive-definite symmetric matrix because the null space of matrix $\left[\underline{A}_{m, n}\right]$ is reduced to $\{0\}$. Since $\left[\underline{B}_{n}\right]$ belongs to $M_{n}^{+}(\mathbb{R})$, matrix $\left[\underline{B}_{n}\right]$ is diagonalizable and can be written as

$$
\left[\underline{B}_{n}\right]=\left[\underline{\Phi}_{n}\right]\left[\underline{\Sigma}_{n}\right]\left[\underline{\Phi}_{n}\right]^{T}
$$

in which $\left[\underline{\Sigma}_{n}\right] \in \mathbb{M}_{n}^{+}(\mathbb{R})$ is the diagonal matrix of the positive eigenvalues of matrix $\left[\underline{B}_{n}\right]$ and where $\left[\underline{\Phi}_{n}\right] \in M_{n}(\mathbb{R})$ is the matrix of the corresponding eigenvectors which is an orthogonal matrix, that is to say such that $\left[\underline{\Phi}_{n}\right]\left[\underline{\Phi}_{n}\right]^{T}=\left[\underline{\Phi}_{n}\right]^{T}\left[\underline{\Phi}_{n}\right]=\left[I_{n}\right]$. Consequently, $\left[\underline{\Sigma}_{n}\right]^{1 / 2}$ is the matrix of the singular values of matrix $\left[\underline{A}_{m, n}\right]$ and we define the matrix $\left[\underline{T}_{n}\right]$ by

$$
\left[\underline{T}_{n}\right]=\left[\underline{B}_{n}\right]^{1 / 2}=\left[\underline{\Phi}_{n}\right]\left[\underline{\Sigma}_{n}\right]^{1 / 2}\left[\underline{\Phi}_{n}\right]^{T} \in \mathbb{M}_{n}^{+}(\mathbb{R})
$$

Since matrix $\left[\underline{T}_{n}\right]$ belongs to $M_{n}^{+}(\mathbb{R})$, it is invertible and the matrix $\left[\underline{U}_{m, n}\right]$ in the decomposition $\left[\underline{A}_{m, n}\right]=\left[\underline{U}_{m, n}\right]\left[\underline{T}_{n}\right]$ can then be constructed by the following equation,

$$
\left[\underline{U}_{m, n}\right]=\left[\underline{A}_{m, n}\right]\left[\underline{T}_{n}\right]^{-1} \in M_{m, n}(\mathbb{R})
$$

The construction of the decomposition is then complete. It should be noted that $\left[\underline{U}_{m, n}\right]^{T}\left[\underline{U}_{m, n}\right]=$ $\left[\underline{T}_{n}\right]^{-T}\left[\underline{A}_{m, n}\right]^{T}\left[\underline{A}_{m, n}\right]\left[\underline{T}_{n}\right]^{-1}=\left[\underline{T}_{n}\right]^{-T}\left[\underline{B}_{n}\right]\left[\underline{T}_{n}\right]^{-1}=\left[\underline{B}_{n}\right]^{-1 / 2}\left[\underline{B}_{n}\right]\left[\underline{B}_{n}\right]^{-1 / 2}=\left[I_{n}\right]$. 


\subsubsection{Definition of ensemble $\mathrm{SE}_{\mathrm{inv}}$}

Let $\left[\underline{A}_{m, n}\right]$ be a rectangular real matrix in $M_{m, n}(\mathbb{R})$ with a null space reduced to $\{0\}$ whose decomposition (see Section 2.5.1) is

$$
\left[\underline{A}_{m, n}\right]=\left[\underline{U}_{m, n}\right]\left[\underline{T}_{n}\right],
$$

in which $\left[\underline{U}_{m, n}\right] \in \mathbb{M}_{m, n}(\mathbb{R})$ and $\left[\underline{T}_{n}\right] \in \mathbb{M}_{n}^{+}(\mathbb{R})$. Since symmetric matrix $\left[\underline{T}_{n}\right]$ is positive definite, there is an upper triangular matrix $\left[\underline{L}_{T_{n}}\right]$ in $\mathbb{M}_{n}(\mathbb{R})$ such that

$$
\left[\underline{T}_{n}\right]=\left[\underline{L}_{T_{n}}\right]^{T}\left[\underline{L}_{T_{n}}\right]
$$

which corresponds to the Cholesky factorization of matrix $\left[\underline{T}_{n}\right]$. A random rectangular matrix $\left[\mathbf{A}_{m, n}\right]$ belonging to the ensemble $\mathrm{SE}_{\mathrm{inv}}$, is a random matrix defined on probability space $(\mathcal{A}, \mathcal{T}, P)$, with values in $M_{m, n}(\mathbb{R})$, whose mean value is

$$
E\left\{\left[\mathbf{A}_{m, n}\right\}=\left[\underline{A}_{m, n}\right] \in \mathbb{M}_{m, n}(\mathbb{R})\right.
$$

and which is written as

$$
\left[\mathbf{A}_{m, n}\right]=\left[\underline{U}_{m, n}\right]\left[\mathbf{T}_{n}\right],
$$

in which the matrix $\left[\underline{U}_{m, n}\right] \in \mathbb{M}_{m, n}(\mathbb{R})$ is such that

$$
\left[\underline{U}_{m, n}\right]^{T}\left[\underline{U}_{m, n}\right]=\left[I_{n}\right],
$$

and where the random matrix $\left[\mathbf{T}_{n}\right]$ with values in $\mathbb{M}_{n}^{+}(\mathbb{R})$ is written as

$$
\left[\mathbf{T}_{n}\right]=\left[\underline{L}_{T_{n}}\right]^{T}\left[\mathbf{G}_{n}\right]\left[\underline{L}_{T_{n}}\right],
$$

with $\left[\mathbf{G}_{n}\right]$ a random matrix in ensemble $\mathrm{SG}^{+}$. Finally, from Eqs. (2), (6) and (71), it can be deduced that

$$
E\left\{\left\|\left[\mathbf{T}_{n}\right]\right\|^{2}\right\}=C_{0}<+\infty \quad, \quad E\left\{\left\|\left[\mathbf{T}_{n}\right]^{-1}\right\|^{2}\right\}=C_{1}<+\infty \quad,
$$

in which $C_{0}$ and $C_{1}$ are two finite positice constants. 


\subsubsection{Properties of a random matrix in ensemble $\mathrm{SE}_{\mathrm{inv}}$}

From Subsections 2.5.1 to 2.5.3, it can easily be deduced that the random matrix $\left[\mathbf{A}_{m, n}\right]$ with values in $M_{m, n}(\mathbb{R})$, belonging to ensemble $\mathrm{SE}_{\text {inv }}$, and defined in Subsection 2.5.3, has the following properties:

(1) Random matrix $\left[\mathbf{A}_{m, n}\right]$ is a second-order random variable and we have the following inequalities,

$$
\begin{gathered}
E\left\{\left\|\left[\mathbf{A}_{m, n}\right]\right\|_{F}^{2}\right\}=E\left\{\left\|\left[\mathbf{T}_{n}\right]\right\|_{F}^{2}\right\}<+\infty, \\
0<C_{2} \leq E\left\{\left\|\left[\mathbf{A}_{m, n}\right]\right\|^{2}\right\} \leq C_{3}<+\infty,
\end{gathered}
$$

in which $C_{2}=\frac{1}{C_{1}}\left\|\left[\underline{U}_{m, n}\right]\right\|^{2}$ and $C_{3}=C_{0}\left\|\left[\underline{U}_{m, n}\right]\right\|^{2}$ with $C_{0}$ and $C_{1}$ are the constants defined in Eq. (72).

(2) The mean value of random matrix $\left[\mathbf{A}_{m, n}\right]$ is such that

$$
E\left\{\left[\mathbf{A}_{m, n}\right]\right\}=\left[\underline{A}_{m, n}\right] \in \mathbb{M}_{m, n}(\mathbb{R})
$$

(3) The left pseudo-inverse $\left[\mathbf{A}_{m, n}\right]^{-1 \ell} \in \mathbb{M}_{n, m}(\mathbb{R})$ of random rectangular matrix $\left[\mathbf{A}_{m, n}\right]$ is defined by

$$
\left[\mathbf{A}_{m, n}\right]^{-1 \ell}=\left\{\left[\mathbf{A}_{m, n}\right]^{T}\left[\mathbf{A}_{m, n}\right]\right\}^{-1}\left[\mathbf{A}_{m, n}\right]^{T} \in \mathbb{M}_{n, m}(\mathbb{R})
$$

Since $\left[\mathbf{A}_{m, n}\right]^{-1 \ell}=\left[\mathbf{T}_{n}\right]^{-1}\left[\underline{U}_{m, n}\right]^{T}$, we deduce that

$$
E\left\{\left\|\left[\mathbf{A}_{m, n}\right]^{-1 \ell}\right\|^{2}\right\} \leq C_{1}\left\|\left[\underline{U}_{m, n}\right]^{T}\right\|^{2}<+\infty
$$

in which $C_{1}$ is the constant defined in Eq. (72).

(4) As previously, the dispersion of random matrix $\left[\mathbf{A}_{m, n}\right]$ is controlled by $\delta_{A}$ such that

$$
\delta_{A}=\left\{\frac{1}{n} E\left\{\left\|\left[\mathbf{G}_{n}\right]-\left[I_{n}\right]\right\|_{F}^{2}\right\}\right\}^{1 / 2},
$$

in which $\delta_{A}$ has to be independent of $n$ and such that $0<\delta_{A}<\sqrt{(n+1)(n+5)^{-1}}$.

\section{Foundations of the nonparametric probabilistic approach of random uncertainties in mechanical systems}

In this part, we explain the main ideas and the foundations of the nonparametric probabilistic approach of data uncertainties and model uncertainties in mechanical systems.

C. Soize - Computer Methods in Applied Mechanics and Engineering (CMAME) (accepted in March 2004) 


\subsection{Mathematical-mechanical modeling of a physical mechanical system}

Let us consider a Physical Mechanical System (PMS). This means that the PMS is the mechanical system for which a predictive model has to be constructed. The mathematical-mechanical modeling of this PMS leads us to a Boundary Value Problem (BVP). The weak formulation of such a BVP introduces several linear operators (such as the mass, damping and stiffness operators of a fixed elastic system occupying a three-dimensional bounded domain). Let $\mathbb{A}_{0}$ be one of these operators which is assumed to be an unbounded operator in a real Hilbert space $\mathbb{H}$ and having an inverse in $\mathbb{H}$. By assumption, the unknown field of this BVP is with values in an admissible space $\mathbb{V}$ which is a Hilbert space such that $\mathbb{V} \subset \mathbb{H}$. It is assumed that the functions belonging to admissible space $\mathbb{V}$ can reasonably approximate any state of the PMS. Let $\mathbb{A}_{\exp }$ be the operator corresponding to $\mathbb{A}_{0}$ for the PMS. This operator is unknown.Only an "approximate" model $\mathbb{A}_{0}$ of $\mathbb{A}_{\exp }$ can be constructed.

\subsection{Mean reduced model}

The reduced model is deduced from the BVP by using the Ritz-Galerkin projection on a finite dimension subspace $\mathbb{H}_{n}$ of $\mathbb{V}$. Let $\left\{\phi_{\alpha}\right\}_{\alpha \geq 1}$ be an Hilbertian basis in $\mathbb{H}$ whose functions $\phi_{\alpha}$ are in space $\mathbb{V}$. Then $\uplus_{n}$ is spanned by $\left\{\phi_{1}, \ldots, \phi_{n}\right\}$ (for instante the eigenmodes). Therefore, the projection of operator $\mathbb{A}_{0}$ on $\mathbb{H}_{n}$ is represented by the real square matrix $\left[A_{0, n}\right]$ in $\mathbb{M}_{n}(\mathbb{R})$. The mean reduced model is defined as the reduced model constructed by using the nominal values of the parameters. Matrix $\left[A_{0, n}\right]$ corresponding to the nominal mean reduced model is rewritten as $\left[\underline{A}_{0, n}\right]$.

\subsection{Updating the mean reduced model using experimental data}

Let $\left[A_{\exp , n}\right]$ be the matrix of the projection of $\mathbb{A}_{\exp }$ on $\uplus_{n}$ (that is to say using the Hibertian basis $\left.\left\{\phi_{\alpha}\right\}_{\alpha \geq 1}\right)$. Therefore, $\left[A_{\exp , n}\right]$ is a matrix in $\mathbb{M}_{n}(\mathbb{R})$ which is assumed to be experimentally identified (indirect experimental identification). The biais $\left[\underline{B}_{0, n}\right]$ between the PMS and the mean reduced model is defined by $\left[\underline{B}_{0, n}\right]=\left[\underline{A}_{0, n}\right]-\left[A_{\exp , n}\right]$. For complex system, it is difficult to develop an accurate predictive model and consequently, the norm $\left\|\left[\underline{B}_{0, n}\right]\right\|$ of biais $\left[\underline{B}_{0, n}\right]$ is not small. Therefore, the biais has to be reduced in updating the nominal parameters of the model. The model updating yields the updated matrix $\left[\underline{A}_{n}\right]$ in $\mathbb{M}_{n}(\mathbb{R})$ of nominal matrix $\left[\underline{A}_{0, n}\right]$. The biais $\left[\underline{B}_{n}\right]=\left[\underline{A}_{n}\right]-\left[A_{\exp , n}\right]$ has a norm $\left\|\left[\underline{B}_{n}\right]\right\|<\left\|\left[\underline{B}_{0, n}\right]\right\|$ which is generally not sufficiently small. Then, a probabilistic model of uncertainties has to be introduced. 


\subsection{Parametric probabilistic approach}

The parametric probabilistic model consists in introducing random variables and stochastic fields as parameters in the BVP in order to model data uncertainties. The statistical reduction methods (such as the truncated Karhunen-Loeve of stochastic fields (see for instance [12])) allow the stochastic reduced model to be constructed. In such a stochastic reduced model, $\left[\underline{A}_{n}\right]$ becomes a random matrix $\left[A_{n}(\mathbf{X})\right]$ in which $\mathbf{X}$ is an $\mathbb{R}^{m}$-valued random variable whose support $\mathcal{D}_{m}$ of its probability measure (probability distribution) $P_{\mathbf{X}}$ is such that $\mathcal{D}_{m} \subset \mathbb{R}^{m}$ and where $\mathbf{x} \mapsto\left[A_{n}(\mathbf{x})\right]$ is a mapping from $\mathbb{R}^{m}$ into $\mathbb{M}_{n}(\mathbb{R})$. Clearly, the range of mapping $\mathbf{x} \mapsto\left[A_{n}(\mathbf{x})\right]$ is a subset $\mathbb{S}_{\text {par }, n}$ of $\mathbb{S}_{n}$ such that

$$
\mathbb{S}_{\text {par }, n} \subset \mathbb{S}_{n} \subset \mathbb{M}_{n}(\mathbb{R})
$$

in which $\mathbb{S}_{n}$ is a subset of $M_{n}(\mathbb{R})$ for which any matrix in $\mathbb{S}_{n}$ is assumed to be invertible in order to simplify the developments. For instance, subset $\mathbb{S}_{n}$ will be $\mathbb{S}_{n}=\mathbb{M}_{n}^{+}(\mathbb{R})$. It should be noted that, if it can be assumed that $\left[A_{\exp , n}\right]$ is surely in $\mathbb{S}_{n}$, then due to random uncertainties, $\left[A_{\exp , n}\right]$ is generally not in $\mathbb{S}_{\mathrm{par}, n}$. Let $\left[\mathbf{A}_{\mathrm{par}, n}\right]$ be the random matrix with values in $\mathbb{M}_{n}(\mathbb{R})$ corresponding to the parametric probabilistic model of data uncertainties and defined by

$$
\left[\mathbf{A}_{\mathrm{par}, n}\right]=\left[A_{n}(\mathbf{X})\right]
$$

Then, the mean-square error between the parametric probabilistic model of random uncertainties and the experimental data is given by

$$
\begin{aligned}
E\left\{\left\|\left[\mathbf{A}_{\mathrm{par}, n}\right]^{-1}-\left[A_{\mathrm{exp}, n}\right]^{-1}\right\|^{2}\right\} & =\int_{\mathcal{D}_{m}}\left\|\left[A_{n}(\mathbf{x})\right]^{-1}-\left[A_{\mathrm{exp}, n}\right]^{-1}\right\|^{2} P_{\mathbf{X}}(d \mathbf{x}) \\
& =\int_{\mathbb{S p a r}, n}\left\|\left[a_{n}\right]^{-1}-\left[A_{\exp , n}\right]^{-1}\right\|^{2} P_{\operatorname{par}}\left(\widetilde{d} a_{n}\right),
\end{aligned}
$$

in which $\widetilde{d} a_{n}$ is the volume element on set $\mathbb{S}_{\text {par, } n}$ and where $P_{\text {par }}$ is the probability measure on $\mathbb{S}_{\text {par }, n}$ defined as the image of $P_{\mathbf{X}}$ on $\mathcal{D}_{m}$ by the mapping $\mathbf{x} \mapsto\left[A_{n}(\mathbf{x})\right]$ and which is such that

$$
E\left\{\left\|\left[\mathbf{A}_{\mathrm{par}, n}\right]^{-1}\right\|^{2}\right\}=\int_{\mathcal{D}_{m}}\left\|\left[A_{n}(\mathbf{x})\right]^{-1}\right\|^{2} P_{\mathbf{X}}(d \mathbf{x})=\int_{\mathbb{S}_{\mathrm{par}, n}}\left\|\left[a_{n}\right]^{-1}\right\|^{2} P_{\mathrm{par}}\left(\widetilde{d} a_{n}\right)<+\infty .
$$

In general, $\mathcal{D}_{m}$ and probability measure $P_{\mathbf{X}}$ on $\mathcal{D}_{m}$ are given. Due to the model uncertainties, the mean-square error defined by Eq. (81) is generally not sufficiently small. 


\subsection{Introduction of the nonparametric probabilistic approach}

The problem is then to introduce a nonparametric probabilistic approach of data and model uncertainties allowing the mean-square error defined by Eq. (81) to be reduced. The nonparametric probabilistic approach of random uncertainties consists in substituting random matrix $\left[\mathbf{A}_{\text {par }, n}\right]$ by a random matrix $\left[\mathbf{A}_{\text {nonpar }, n}\right]$ such that

$$
E\left\{\left[\mathbf{A}_{\text {nonpar }, n}\right]\right\}=\left[\underline{A}_{n}\right] \quad, \quad E\left\{\left\|\left[\mathbf{A}_{\text {nonpar }, n}\right]^{-1}\right\|^{2}\right\}<+\infty \quad .
$$

The probability measure $P_{\text {nonpar }}$ on $\mathbb{S}_{n}$ of random matrix $\left[\mathbf{A}_{\text {nonpar, } n}\right]$ is then directly constructed by using the random matrix theory (see Section 2). Then, the mean-square error between the nonparametric probabilistic model of random uncertainties and the experimental data is given by

$$
E\left\{\left\|\left[\mathbf{A}_{\text {nonpar }, n}\right]^{-1}-\left[A_{\text {exp }, n}\right]^{-1}\right\|^{2}\right\}=\int_{\mathbb{S}_{n}}\left\|\left[a_{n}\right]^{-1}-\left[A_{\text {exp }, n}\right]^{-1}\right\|^{2} P_{\text {nonpar }}\left(\widetilde{d} a_{n}\right),
$$

in which $\widetilde{d} a_{n}$ is the volume element on set $\mathbb{S}_{n}$.

\subsection{Capability of the nonparametric probabilistic approach}

Since $\mathbb{S}_{\text {par }, n} \subset \mathbb{S}_{n}$, we can take $P_{\text {nonpar }}=P_{\text {par }}$ in which the support of probability measure is $\mathbb{S}_{\text {par }, n}$. In this case, we have $\left[\mathbf{A}_{\text {nonpar }, n}\right]=\left[\mathbf{A}_{\text {par, }, n}\right]$ which proves that the nonparametric model has the capability to take into account data uncertainties. In addition, since the support of $P_{\text {nonpar }}$ is $\mathbb{S}_{n}$ with $\mathbb{S}_{\mathrm{par}, n} \subset \mathbb{S}_{n}$, the nonparametric model allows a larger class of random matrices to be constructed and consequently, has, a priori, the capability to take into account the model uncertainties.

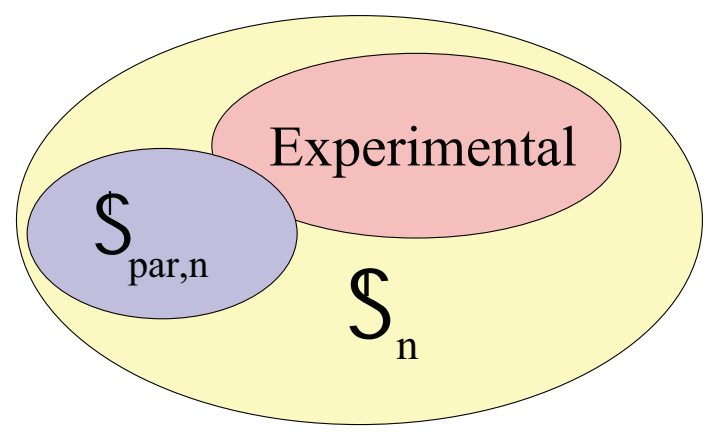

Fig. 4. Set $\mathbb{S}_{\mathrm{par}, \mathrm{n}}$ of the values of random matrices $\left[\mathbf{A}_{\mathrm{par}, \mathrm{n}}\right]$ for the parametric approach, set $\mathbb{S}_{\mathrm{n}}$ of values of random matrices $\left[\mathbf{A}_{\text {nonpar, }}\right]$ for the nonparametric approach, set of matrices $\left[A_{\text {exper,n }}\right]$ for experimental physical system.

For instance, let us assume that the model uncertainties are sufficiently high for that $\left[A_{\exp , n}\right] \notin \mathbb{S}_{\text {par }, n}$ but, as we explained above, we have $\left[A_{\exp , n}\right] \in \mathbb{S}_{n}$ with $\mathbb{S}_{\text {par, }, n} \subset \mathbb{S}_{n}$ (see Fig. 4). Equation (81) 
shows that the mean-square error cannot be reduced even if probability measure $P_{\text {par }}$ could be arbitrary chosen. On the other hand, since $\left[A_{\exp , n}\right]$ belongs to $\mathbb{S}_{n}$, Eq. (84) shows that there are probability measures $P_{\text {nonpar }}$ on $\mathbb{S}_{n}$ which allow the mean-square error to be reduced. For instance, if $\delta_{0}$ is the Dirac measure on $\mathbb{M}_{n}(\mathbb{R})$, then the probability measure $P_{\text {nonpar }}\left(\widetilde{d} a_{n}\right)=\delta_{0}\left(\left[a_{n}\right]-\left[A_{\exp , n}\right]\right)$ leads the error to be zero because $\left[A_{\exp , n}\right]$ belongs to $\mathbb{S}_{n}$. Of course, $P_{\text {nonpar }}$ cannot be arbitrary chosen on $\mathbb{S}_{n}$ but has to be constructed using the matrix theory introduced in Section 2. Consequently and intuitively, there is a probability measure $P_{\text {nonpar }}$ on $\mathbb{S}_{n}$ verifying the properties defined by Eq. (83), such that

$$
\int_{\mathbb{S}_{n}}\left\|\left[a_{n}\right]^{-1}-\left[A_{\text {exp }, n}\right]^{-1}\right\|^{2} P_{\text {nonpar }}\left(\widetilde{d} a_{n}\right)<\int_{\mathbb{S p a r}, n}\left\|\left[a_{n}\right]^{-1}-\left[A_{\text {exp }, n}\right]^{-1}\right\|^{2} P_{\text {par }}\left(\widetilde{d} a_{n}\right)
$$

which means that

$$
E\left\{\left\|\left[\mathbf{A}_{\text {nonpar }, n}\right]^{-1}-\left[A_{\exp , n}\right]^{-1}\right\|^{2}\right\}<E\left\{\left\|\left[\mathbf{A}_{\text {par }, n}\right]^{-1}-\left[A_{\exp , n}\right]^{-1}\right\|^{2}\right\} \quad .
$$

\section{Nonparametric model of random uncertainties for linear and nonlinear transient dynamics}

In this part, for complex dynamical systems, we summarize the nonparametric probabilistic approach of data uncertainties and model uncertainties, that we introduced in [27-33] and for which the foundations have been given in Section 3. The continuous aspects are presented in [29]. In order to limit the developments, the presentation will be limited to the discrete cases.

\subsection{Introduction of the mean finite element model}

We consider a nonlinear dynamic system constituted of a three-dimensional damped fixed structure around a static equilibrium configuration considered as a natural state without prestresses and subjected to an external load. The basic finite element model of this nonlinear dynamic system is called the "mean finite element model" (the underlined quantities refer to this "mean finite element model") and leads to the following nonlinear differential equation,

$$
[\underline{\mathbb{M}}] \underline{\mathbf{y}}(t)+[\underline{\mathbb{D}}] \underline{\dot{\mathbf{y}}}(t)+[\underline{\mathbb{K}}] \underline{\mathbf{y}}(t)+\mathbf{f}_{\mathrm{NL}}(\underline{\mathbf{y}}(t), \underline{\mathbf{y}}(t))=\mathbf{f}(t)
$$

in which $\underline{\mathbf{y}}=\left(\underline{y}_{1}, \ldots, \underline{y}_{m}\right)$ is the unknown time response vector of the $m$ DOFs (displacements and/or rotations); $\underline{\mathbf{y}}$ and $\underline{\ddot{\mathbf{y}}}$ are the velocity and acceleration vectors respectively; $\mathbf{f}(t)=$ 
$\left(f_{1}(t), \ldots, f_{m}(t)\right)$ is the known external load vector of the $m$ inputs (forces and/or moments); [ $\left.\underline{\mathbb{M}}\right]$, $[\underline{\mathbb{D}}]$ and $[\underline{\mathbb{K}}]$ are the mass, damping and stiffness matrices of the linear part of the model, which are positive-definite symmetric $(m \times m)$ real matrices; $(\mathbf{y}, \mathbf{z}) \mapsto \mathbf{f}_{\mathrm{NL}}(\mathbf{y}, \mathbf{z})$ is a nonlinear mapping from $\mathbb{R}^{m} \times \mathbb{R}^{m}$ into $\mathbb{R}^{m}$ modeling additional nonlinear damping and restoring forces such that $\mathbf{f}_{\mathrm{NL}}(0,0)=0$. The linear case can be derived from Eq. (87) in taking $\mathbf{f}_{\mathrm{NL}}=0$.

\subsection{Introduction of the mean reduced model}

The generalized eigenvalue problem associated with the mean mass and stiffness matrices of the mean finite element model is written as $[\underline{K}] \underline{\varphi}=\underline{\lambda}[\underline{M}] \underline{\varphi}$. Since $[\underline{\mathbb{K}}]$ is a positive-definite matrix, we have $0<\underline{\lambda}_{1} \leq \underline{\lambda}_{2} \leq \ldots \leq \underline{\lambda}_{m}$ and the associated elastic modes $\left\{\underline{\varphi}_{1}, \underline{\varphi}_{2}, \ldots\right\}$ are such that $\left\langle[\underline{\mathbb{M}}] \underline{\varphi}_{\alpha}, \underline{\varphi}_{\beta}>=\underline{\mu}_{\alpha} \delta_{\alpha \beta}\right.$ and $<[\underline{\mathbb{K}}] \underline{\varphi}_{\alpha}, \underline{\varphi}_{\beta}>=\underline{\mu}_{\alpha} \underline{\omega}_{\alpha}^{2} \delta_{\alpha \beta}$ in which $\underline{\omega}_{\alpha}=\sqrt{\underline{\lambda}_{\alpha}}$ is the eigenfrequency of elastic mode $\underline{\varphi}_{\alpha}$ whose normalization is defined by the generalized mass $\underline{\mu}_{\alpha}$. The mean reduced model of the dynamic system whose mean finite element model is defined by Eq. (87) is obtained in constructing the projection of the mean finite element model on the subspace $H_{n}$ of $\mathbb{R}^{m}$ spanned by $\left\{\underline{\varphi}_{1}, \ldots, \underline{\varphi}_{n}\right\}$ with $n \ll m$. Let $\left[\underline{\Phi}_{n}\right]$ be the $(m \times n)$ real matrix whose columns are vectors $\left\{\underline{\varphi}_{1}, \ldots, \underline{\varphi}_{n}\right\}$. The generalized force $\mathbf{F}^{n}(t)$ is an $\mathbb{R}^{n}$-vector such that $\mathbf{F}^{n}(t)=\left[\underline{\Phi}_{n}\right]^{T} \mathbf{f}(t)$. The generalized mass, damping and stiffness matrices $\left[\underline{M}_{n}\right],\left[\underline{D}_{n}\right]$ and $\left[\underline{K}_{n}\right]$ are positive-definite symmetric $(n \times n)$ real matrices such that $\left[\underline{M}_{n}\right]_{\alpha \beta}=\underline{\mu}_{\alpha} \delta_{\alpha \beta},\left[\underline{D}_{n}\right]_{\alpha \beta}=<[\underline{\mathbb{D}}] \underline{\varphi}_{\beta}, \underline{\varphi}_{\alpha}>$ and $\left[\underline{K}_{n}\right]_{\alpha \beta}=\underline{\mu}_{\alpha} \underline{\omega}_{\alpha}^{2} \delta_{\alpha \beta}$, in which, generally, $\left[\underline{D}_{n}\right]$ is a full matrix. Consequently, the mean reduced model of the nonlinear dynamic system is written as the projection $\underline{\mathbf{y}}^{n}$ of $\underline{\mathbf{y}}$ on $H_{n}$ can be written as $\underline{\mathbf{y}}^{n}(t)=\left[\underline{\Phi}_{n}\right] \underline{\mathbf{q}}^{n}(t)$ in which the vector $\underline{\mathbf{q}}^{n}(t) \in \mathbb{R}^{n}$ of the generalized coordinates verifies the mean nonlinear differential equation,

$$
\left.\left[\underline{M}_{n}\right] \underline{\ddot{\mathbf{q}}}^{n}(t)+\left[\underline{D}_{n}\right] \underline{\dot{\mathbf{q}}}^{n}(t)+\left[\underline{K}_{n}\right] \underline{\mathbf{q}}^{n}(t)+\mathbf{F}_{\mathrm{NL}}^{n} \underline{\mathbf{q}}^{n}(t), \underline{\dot{\mathbf{q}}}^{n}(t)\right)=\mathbf{F}^{n}(t), \forall t \geq 0,
$$

where, for all $\mathbf{q}$ and $\mathbf{p}$ in $\mathbb{R}^{n}$,

$$
\mathbf{F}_{\mathrm{NL}}^{n}(\mathbf{q}, \mathbf{p})=\left[\underline{\Phi}_{n}\right]^{T} \mathbf{f}_{\mathrm{NL}}\left(\left[\underline{\Phi}_{n}\right] \mathbf{q},\left[\underline{\Phi}_{n}\right] \mathbf{p}\right)
$$

\subsection{Nonparametric probabilistic approach of random uncertainties}

The principle of construction of the nonparametric probabilistic approach of random uncertainties for the linear and nonlinear dynamic systems whose mean finite element model is defined by 
Eq. (87), is given in Section 3. It consists in substituting the generalized mass, damping and stiffness matrices of the mean reduced model (see Eq. (88)) by random matrices $\left[\mathbf{M}_{n}\right],\left[\mathbf{D}_{n}\right]$ and $\left[\mathbf{K}_{n}\right]$. If the nonlinear forces are uncertain, a usual parametric model can be used for these nonlinear forces. In this case, a nonparametric-parametric mixed formulation can be constructed (see [5]).

The construction of the probability model of random matrices $\left[\mathbf{M}_{n}\right],\left[\mathbf{D}_{n}\right]$ and $\left[\mathbf{K}_{n}\right]$ defined on probability space $(\mathcal{A}, \mathcal{T}, \mathcal{P})$, is based on the available information deduced from the fundamental properties of a dynamical system and from additional properties required in order that a secondorder stochastic solution exists (see [27,28]). It can then be deduced that random matrices $\left[\mathbf{M}_{n}\right]$, $\left[\mathbf{D}_{n}\right]$ and $\left[\mathbf{K}_{n}\right]$ have to be such that

$$
\begin{gathered}
{\left[\mathbf{M}_{n}\right],\left[\mathbf{D}_{n}\right],\left[\mathbf{K}_{n}\right] \in \mathbb{M}_{n}^{+}(\mathbb{R}) \text { a.s. },} \\
E\left\{\left[\mathbf{M}_{n}\right]\right\}=\left[\underline{M}_{n}\right], E\left\{\left[\mathbf{D}_{n}\right]\right\}=\left[\underline{D}_{n}\right], E\left\{\left[\mathbf{K}_{n}\right]\right\}=\left[\underline{K}_{n}\right], \\
E\left\{\left\|\left[\mathbf{M}_{n}\right]^{-1}\right\|_{F}^{2}\right\}<+\infty, E\left\{\left\|\left[\mathbf{D}_{n}\right]^{-1}\right\|_{F}^{2}\right\}<+\infty, E\left\{\left\|\left[\mathbf{K}_{n}\right]^{-1}\right\|_{F}^{2}\right\}<+\infty,
\end{gathered}
$$

Since matrices $\left[\underline{M}_{n}\right],\left[\underline{D}_{n}\right]$ and $\left[\underline{K}_{n}\right]$ are in $M_{n}^{+}(\mathbb{R})$, there are upper triangular matrices $\left[\underline{L}_{M_{n}}\right]$, $\left[\underline{L}_{D_{n}}\right]$ and $\left[\underline{L}_{K_{n}}\right]$ in $\mathbb{M}_{n}(\mathbb{R})$ such that

$$
\left[\underline{M}_{n}\right]=\left[\underline{L}_{M_{n}}\right]^{T}\left[\underline{L}_{M_{n}}\right] \quad, \quad\left[\underline{D}_{n}\right]=\left[\underline{L}_{D_{n}}\right]^{T}\left[\underline{L}_{D_{n}}\right] \quad, \quad\left[\underline{K}_{n}\right]=\left[\underline{L}_{K_{n}}\right]^{T}\left[\underline{L}_{K_{n}}\right]
$$

From Sections 2.2.2 and 2.2.4, we deduce that random matrices $\left[\mathbf{M}_{n}\right],\left[\mathbf{D}_{n}\right]$ and $\left[\mathbf{K}_{n}\right]$ have to be in ensemble $\mathrm{SE}^{+}$and are independent, each one being a random matrix for which the probability model in defined in Section 2.2.1. Consequently, we have (see Eqs. (30)-(32)),

$$
\left[\mathbf{M}_{n}\right]=\left[\underline{L}_{M_{n}}\right]^{T}\left[\mathbf{G}_{M_{n}}\right]\left[\underline{L}_{M_{n}}\right],\left[\mathbf{D}_{n}\right]=\left[\underline{L}_{D_{n}}\right]^{T}\left[\mathbf{G}_{D_{n}}\right]\left[\underline{L}_{D_{n}}\right],\left[\mathbf{K}_{n}\right]=\left[\underline{L}_{K_{n}}\right]^{T}\left[\mathbf{G}_{K_{n}}\right]\left[\underline{L}_{K_{n}}\right]
$$

in which random matrices $\left[\mathbf{G}_{M_{n}}\right],\left[\mathbf{G}_{D_{n}}\right]$ and $\left[\mathbf{G}_{K_{n}}\right]$ are in ensemble $\mathrm{SG}^{+}$defined in Section 2.1. The parameters $\delta_{M}, \delta_{D}$ and $\delta_{K}$ allowing the dispersion of random matrices $\left[\mathbf{M}_{n}\right],\left[\mathbf{D}_{n}\right]$ and $\left[\mathbf{K}_{n}\right]$ to be controlled are defined by Eq. (37) and have to verify Eq. (38). The probability distribution of each random matrix $\left[\mathbf{G}_{M_{n}}\right],\left[\mathbf{G}_{D_{n}}\right]$ or $\left[\mathbf{G}_{K_{n}}\right]$ is defined in Section 2.1.3 and its algebraic representation is defined in Section 2.1.7.

The stochastic transient response of the nonlinear dynamic system with the nonparametric probabilistic approach of random uncertainties, whose mean reduced model is defined by Eq. (88), is the 
stochastic process $\mathbf{Y}^{n}(t)$, indexed by $\mathbb{R}^{+}$, with values $\mathbb{R}^{m}$, such that $\mathbf{Y}^{n}(t)=\left[\underline{\Phi}_{n}\right] \mathbf{Q}^{n}(t)$ in which the stochastic process $\mathbf{Q}^{n}(t)$, indexed by $\mathbb{R}^{+}$, with values $\mathbb{R}^{n}$, is such that

$$
\left[\mathbf{M}_{n}\right] \ddot{\mathbf{Q}}^{n}(t)+\left[\mathbf{D}_{n}\right] \dot{\mathbf{Q}}^{n}(t)+\left[\mathbf{K}_{n}\right] \mathbf{Q}^{n}(t)+\mathbf{F}_{\mathrm{NL}}^{n}\left(\mathbf{Q}^{n}(t), \dot{\mathbf{Q}}^{n}(t)\right)=\mathbf{F}^{n}(t), \forall t \geq 0,
$$

with the initial conditions, $\mathbf{Q}^{n}(0)=0$ and $\dot{\mathbf{Q}}^{n}(0)=0$.

\subsection{Stochastic solution as a second-order stochastic process}

For any $T>0$, it is proved (see [30]) that, under reasonable assumptions concerning the nonlinear damping and restoring forces, and if $\int_{0}^{T}\|\mathbf{f}(t)\|^{2} d t<+\infty$, then for all $t$ in $[0, T]$, we have

$$
E\left\{\left\|\mathbf{Y}^{n}(t)\right\|^{2}\right\} \leq C_{1}<+\infty \quad, \quad E\left\{\left\|\dot{\mathbf{Y}}^{n}(t)\right\|^{2}\right\} \leq C_{2}<+\infty
$$

in which $C_{1}$ and $C_{2}$ are positive constants that are independent of $n$ and $t$.

\subsection{Construction of the stochastic solution}

The stochastic solution of Eq. (95) is constructed using the Monte Carlo numerical simulation, the realizations of random matrix $\left[\mathbf{A}_{n}\right]$, in which $\left[\mathbf{A}_{n}\right]$ represents random matrices $\left[\mathbf{M}_{n}\right],\left[\mathbf{D}_{n}\right]$ or $\left[\mathbf{K}_{n}\right]$, being constructed by using Eqs. (94) and (23) with properties defined in Section 2.1.7. It should be noted that the numerical cost is low with such a method because Eq. (95) corresponds to a stochastic reduced model with $n \ll m$.

\subsection{Example of transient response of a nonlinear stochastic dynamical system}

In this section, we consider the stochastic transient response of the nonlinear dynamical system studied in [28], corresponding to Eq. (95) and that we present herein below as a simple example for illustrating the nonlinar case. A much more complex dynamical system is analyzed in [5]). The nonlinear dynamical system under consideration is defined in Figure 5 and is composed of a linear thin plane in bending mode with a nonlinearity due to a nonlinear restoring force induced by two stops modeled by high stiffness symmetric barriers which limit the vibration amplitudes of the plate. The plate is rectangular, homogeneous, isotropic, in bending mode, with constant thickness $4 \times 10^{-4} \mathrm{~m}$, width $0.40 \mathrm{~m}$, length $0.50 \mathrm{~m}$, mass density $7800 \mathrm{~kg} / \mathrm{m}^{3}$, Young's modulus $2.1 \times 10^{11} \mathrm{~N} / \mathrm{m}^{2}$ and Poisson ratio 0.29 . This plate is simply supported on 3 edges and free on the fourth edge corresponding to $x_{2}=0$. To this plate are attached one point mass having a mass 
of $4 \mathrm{~kg}$ and one spring having a stiffness coefficient $k=2.388 \times 10^{7} \mathrm{~N} / \mathrm{m}$. Consequently, the dynamical system is not homogeneous. The two stops are located at the free edge. The plate is free between the stops $[-0.002,0.002] \mathrm{m}$ and the stiffness of the two symmetric barriers is $25000 \mathrm{~N} / \mathrm{m}$.

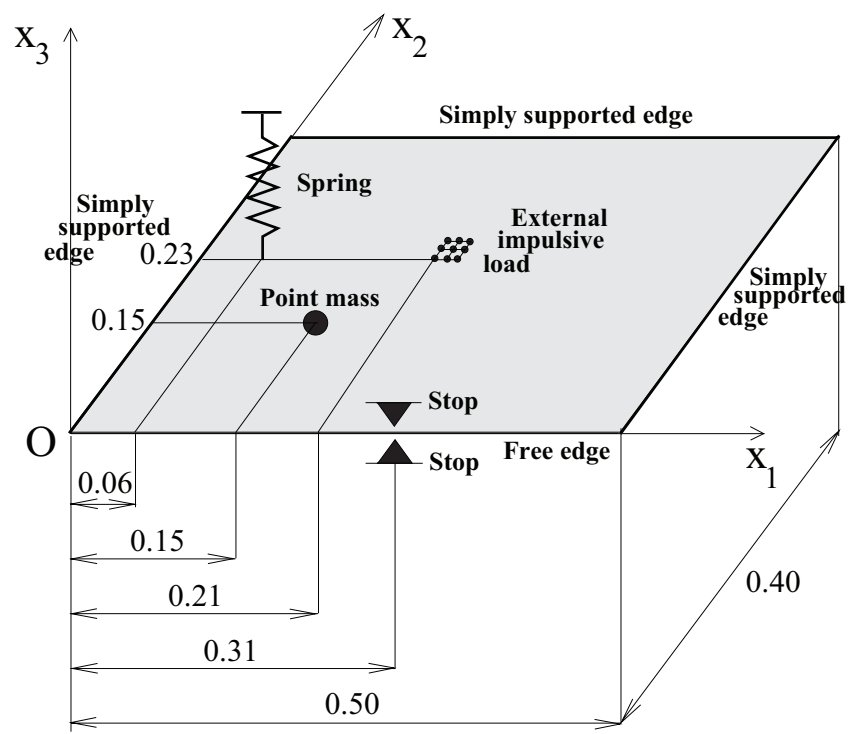

Fig. 5. Geometry of the nonlinear dynamical system

The mean finite element model of the plate is composed of 2000 four-node square plate finite elements and there are $m=6009$ degrees of freedom. The eigenfrequencies calculated with the mean finite element model of the linear plate without the stops are such that $\underline{\nu}_{1}=1.94, \underline{\nu}_{2}=10.28$, $\underline{\nu}_{3}=15.47, \ldots, \underline{\nu}_{8}=53.5, \underline{\nu}_{9}=66.1, \underline{\nu}_{10}=68.9, \ldots, \underline{\nu}_{30}=198.3, \underline{\nu}_{31}=206.0, \underline{\nu}_{32}=208.9$, $\ldots, \underline{\nu}_{50}=330.9, \underline{\nu}_{51}=336.3, \ldots, \underline{\nu}_{100}=670.8, \underline{\nu}_{120}=817.6 \mathrm{~Hz}$. The excitation is an impulsive load defined in Figure 5 whose impulse function is defined in Figure 6.

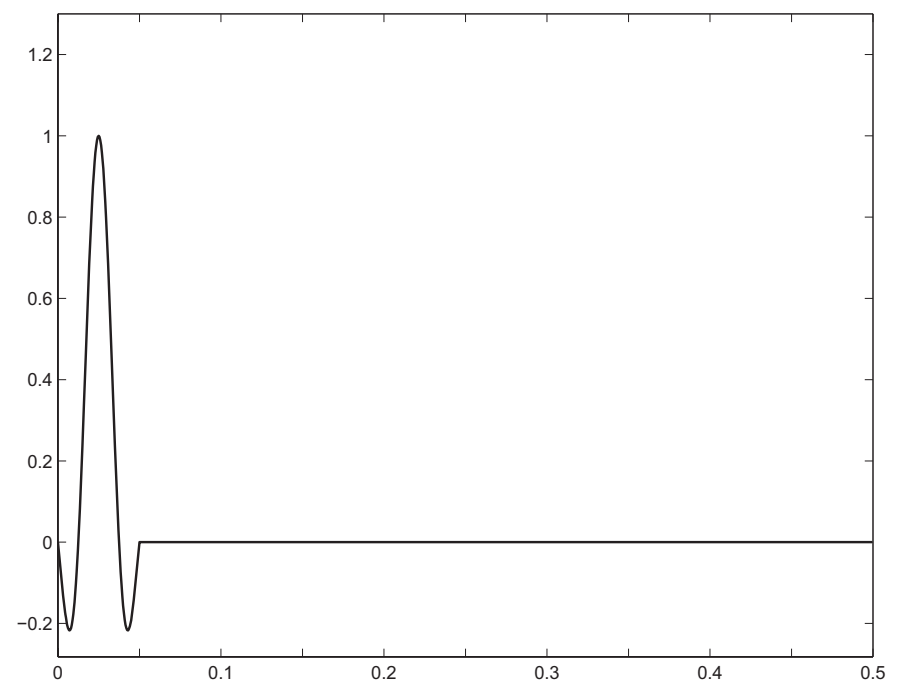

Fig. 6. Graph of wave impulse function as a function of time 
The main part of the energy of this impulse function is distributed over the $[0,60] \mathrm{Hz}$ frequency band in which there are 8 elastic modes for the associated linear underlying dynamical system. The damping matrix $[\underline{\mathbb{D}}]$ of the mean finite element model is written as $[\underline{\mathbb{D}}]=a[\underline{\mathbb{M}}]+b[\underline{\mathbb{K}}]$ in which $a$ and $b$ are defined by $a=2 \underline{\xi} \Omega_{\max } \Omega_{\min } /\left(\Omega_{\max }+\Omega_{\min }\right), b=2 \underline{\xi} /\left(\Omega_{\max }+\Omega_{\min }\right)$ in which $\underline{\xi}=0.04$, $\Omega_{\min }=2 \pi \times 2 \mathrm{rad} / \mathrm{s}$ and $\Omega_{\max }=2 \pi \times 100 \mathrm{rad} / \mathrm{s}$. Below, we are interested in the normalized response spectrum with respect to $g=9.81 \mathrm{~m} / \mathrm{s}^{2}$, over the $[1,200] H z$ frequency band and for the observationDOF corresponding to the normal displacement of the mesh node located at coordinates $x_{1}=0.37, x_{2}=0.15$. The dispersion parameters for the random generalized mass, damping and stiffness matrices are $\delta_{M}=\delta_{D}=\delta_{K}=0.2$. The nonlinear transient response of the dynamical system with random uncertainties is calculated by using the Monte Carlo numerical simulation method with 500 realizations. For given generalized mass, damping and stiffness matrices, the nonlinear evolution problem defined by Eq. (95) is solved by using the Newmark implicit step-bystep integration scheme and an additional numerical iteration procedure for solving the nonlinear algebraic equations at each time step. The value of the time-step size is $\Delta t=1 / 2000 s$ and the number of time steps is 8000 . Convergence with respect to the dimension $n$ of the reduced model can be analyzed in studying the function $n \mapsto\left\|\left|\mathbf{Q}^{n} \|\right|\right.$ in which $\|\left|\mathbf{Q}^{n} \|\right|=\left\{E\left\{\int_{0}^{T}\left\|\mathbf{Q}^{n}(t)\right\|^{2} d t\right\}^{1 / 2}\right.$ is the mean-square norm. Using the usual estimation of the mathematical expectation operator $E$, convergence with respect to the dimension $n$ and the number $n_{s}$ of realizations used in the Monte Carlo numerical method, is studied by constructing the following function,

$$
\operatorname{Conv}\left(n_{s}, n\right)=\left\{\frac{1}{n_{s}} \sum_{k=1}^{n_{s}} \int_{0}^{T}\left\|\mathbf{Q}^{n}\left(t, \theta_{k}\right)\right\|^{2} d t\right\}^{1 / 2}
$$

Figure 7 displays the graphs of functions $n_{s} \mapsto \log _{10}\left\{\operatorname{Conv}\left(n_{s}, n\right)\right\}$ from $\{1,2, \ldots, 500\}$ into $\mathbb{R}$, for $n=10,20,30,50,100$. Convergence with respect to $n$ and $n_{s}$ is obtained for $n=50$ and $n_{s}=300$. Figure 8 corresponds to the transient response of the nonlinear dynamical system with random uncertainties. This figure corresponds to the base 10 logarithm of the random normalized response spectrum for the considered observation (vertical axis) as a function of the base 10 logarithm of the frequency in Hertz for the $[1,200] H z$ frequency band. The mid irregular thin solid line represents the deterministic response of the mean finite element model. The mid smoothed thin solid line represents the mean value of the model with random uncertainties. The lower and upper thick solid lines represent the lower and upper envelopes of the confidence region corresponding to a probability level of 0.95 ; this confidence region is estimated by using the Chebychev inequality. 
The lower and upper thin solid lines correspond to the extreme value statistics. It can be seen that the confidence region gives a good estimation of the extreme value statistics.

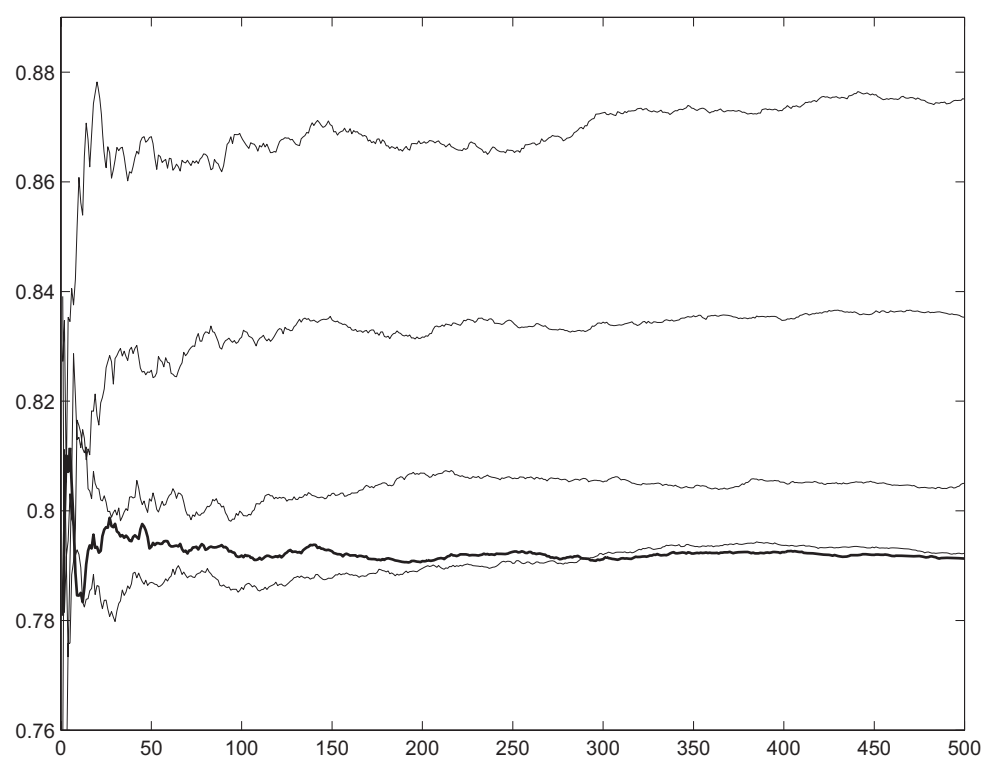

Fig. 7. Convergence in the nonlinear case. Graphs of functions $n_{s} \mapsto \log _{10}\left\{\operatorname{Conv}\left(n_{s}, n\right)\right\}$ for $n=10,20$ and 30 (three upper thin solid lines, for $n=50$ (lower thin solid line) and for $n=100$ (lower thick solid line).

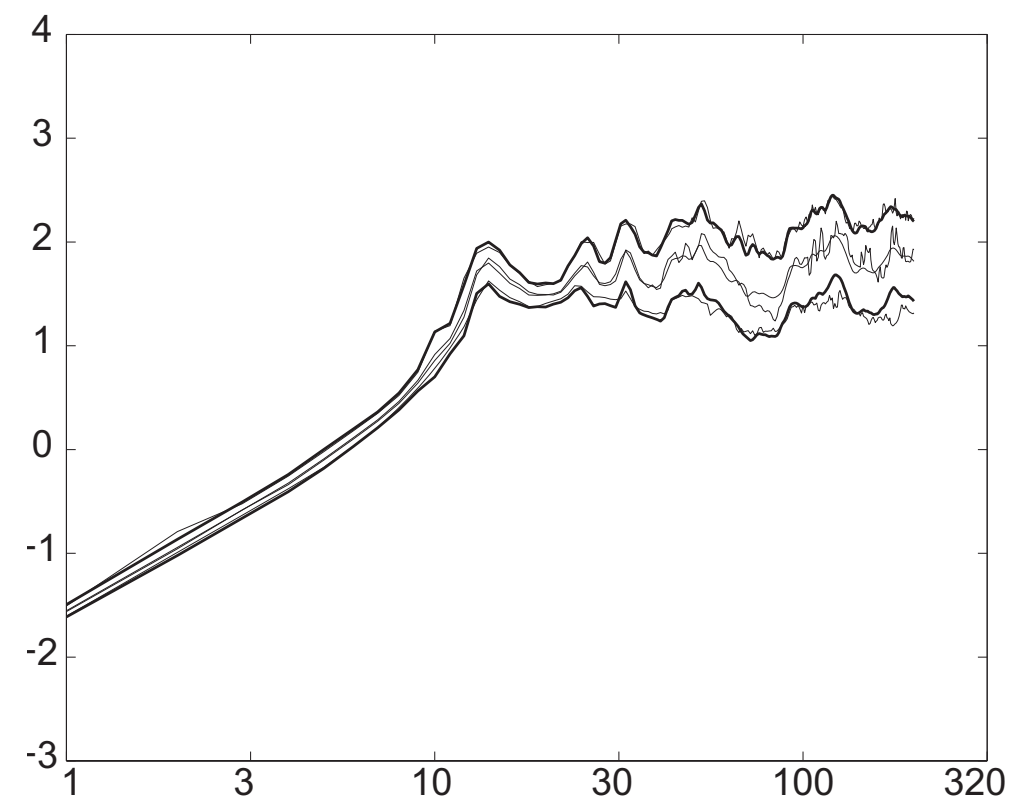

Fig. 8. Random normalized response spectrum in $\mathrm{dB}$ versus frequency in Hertz (log scale). Deterministic response of the mean model (mid irregular thin solid line). Mean value of the model with random uncertainties (mid smoothed thin solid line). Lower and upper envelopes of the confidence region (lower and upper thick solid lines). Extreme value statistics (lower and upper thin solid lines).

For the underlying linear dynamical system, since the energy of the impulse input is concentrated in the $[0,60] \mathrm{Hz}$ frequency band and since there are 8 eigenfrequencies in this frequency band, 
then the dynamics of the transient output is modal type and is concentrated in the same $[0,60] \mathrm{Hz}$ frequency band. For the nonlinear dynamic system, the energy of the impulse input, which is always concentrated in the $[0,60] \mathrm{Hz}$ frequency band, is spread out over the $[0,200] \mathrm{Hz}$ broad frequency band due to the nonlinearity in the dynamical system. This energy is sufficient for exciting the eigenmodes whose eigenfrequencies belong to the $[60,200] \mathrm{Hz}$ frequency band. These modes are sensitive to random uncertainties and it should be noted that the size of the confidence region increases in the $[60,200] \mathrm{Hz}$ frequency band when frequency is increasing. This means that, for the nonlinear dynamic system studied in the example presented, the role plays by random uncertainties increases in the upper part of the frequency band which is not directly excited by the impulse input.

\subsection{Example of transient response of a linear stochastic dynamical system with non homogeneous} random uncertainties and experimental comparison
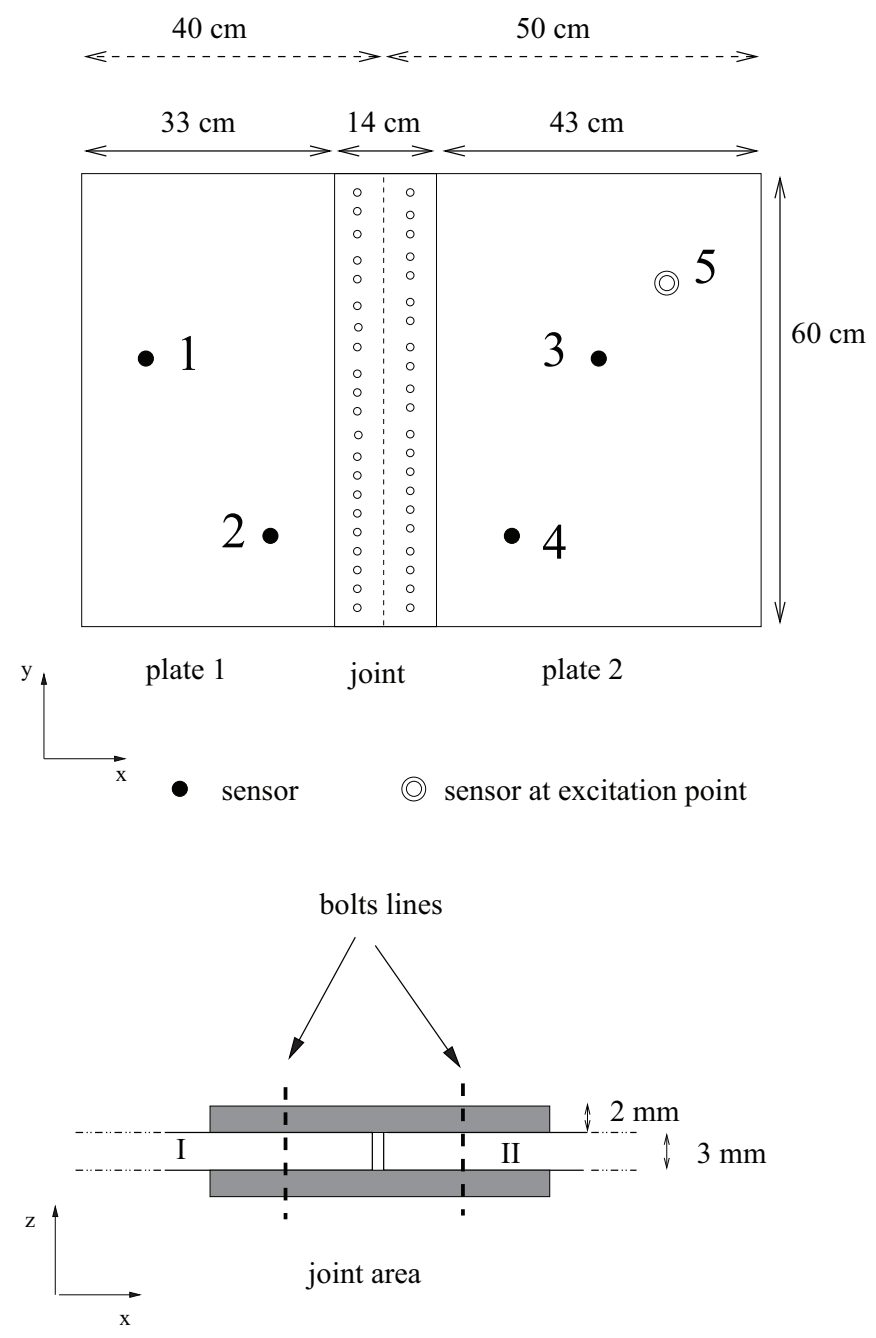

Fig. 9. Geometry of the linear dynamical system 
In this section, we present an example devoted to the transient response of a linear stochastic dynamical system with non homogeneous random uncertainties. A comparison with experimental results is given. All the numerical and experimental results are taken from [6]. The design, the geometry, the dimensions of the plates and of the complex joint, the thicknesses of the plates, the excitation and observation points of the linear dynamical system are defined in Figure 9. This dynamical system is constituted of two Dural plates connected together through a complex joint constituted of 2 smaller Dural plates tightened by 2 lines of 20 bolts. Uncertainties in plates 1 and 2 are very low. In opposite, uncertainties in the complex joint are very high. This is the reason why uncertainties in this dynamical system are non homogeneous. The methodology used for implementing the nonparametric probabilistic approach of random uncertainties in such a dynamical system consists in using a dynamic substructuring method [4], for which every substructure gets its own uncertainty level which is described by using the nonparametric probabilistic approach presented in Section 3 (for the details, see [6]). This dynamical system is in free-free conditions and is excited in bending modes by an impulsive load which is a point force over the $[1000,1200]$ $\mathrm{Hz}$ frequency band, applied to node 5 in plate 2(see Figure 9) and whose experimental signals in frequency and in time domains are displayed in Figure 10.
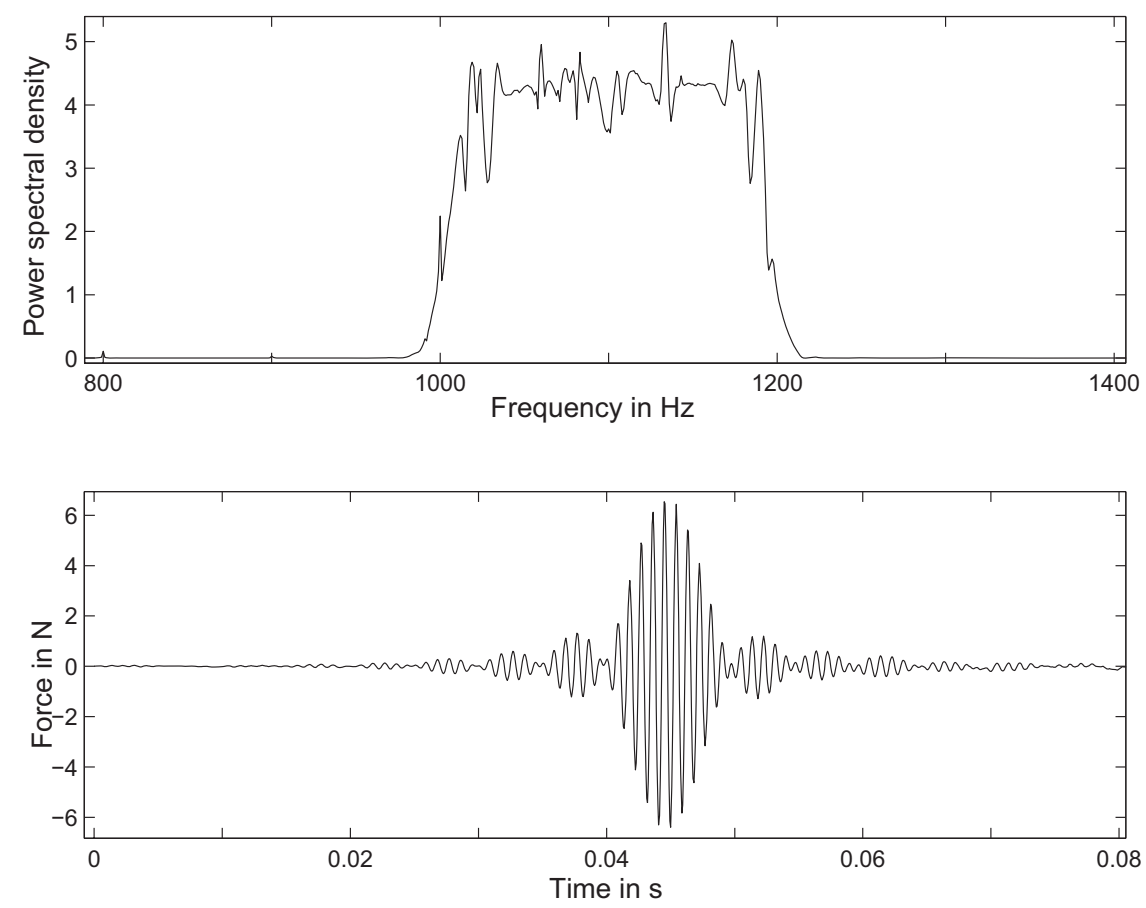

Fig. 10. Impulsive load defined as a point force applied to node 5: frequency domain representation (top figure), time domain representation (down figure). 
Concerning the mean mechanical model, plates 1 and 2 are modeled by homogeneous isotropic thin plates with $0.003 \mathrm{~m}$ and the complex joint is modeled by an equivalent homogeneous orthotropic thin plate with thichness $0.007 \mathrm{~m}$. The mean finite element model of each substructure (plate 1, plate 2 and complex joint) is represented by a uniform finite element model of 4 nodes thin plates elements with 6222 DOFs for plate 1, 8052 DOFs for plate 2 and 2745 DOFs for the complex joint, e.g. a total amount of more than 16000 DOFs. The computation of the global-dynamical-system eigenfrequencies with the mean finite element model yields 65 eigenfrequencies below $1000 \mathrm{~Hz}$ and 13 eigenfrequencies in $[1000,1200] \mathrm{Hz}$ frequency band. The updating of the first 3 elastic modes of the mean finite element model with the experimental results has been performed using the elastic parameters of the orthotropic model of the equivalent plate modeling the complex joint. The damping ratios of the mean finite element model, deduced from the measurements, is 0.0021 .

Let $\delta_{M}, \delta_{D}$ and $\delta_{K}$ be the dispersion parameters of the nonparametric probabilistic approach for mass, damping and stiffness uncertainties of each substructure (plate1, plate 2 and complex joint). The mass of the mean model has been updated with experiments and consequently, there are practically no mass uncertainties in each substructure. Consequently, the mass dispersion parameters for plates 1 and 2 are such that $\delta_{M}^{\text {plates }}=0$ and for the complex joint is such that $\delta_{M}^{C J}=0$. In order to evaluate the role played by the non homogeneity of damping and stiffness uncertainties through the three substructures, which are assumed to be larger in the complex joint than in plates 1 and 2, a sensitivity analysis with respect to the dispersion parameters has been carried out. Since the impulsive load excites the superior elastic modes belonging to [1000,1200] $\mathrm{Hz}$ frequency band, the damping and stiffness model uncertainties are not zero for plates 1 and 2 . Finally, the results displayed below correspond to the following values of the damping and stiffness dispersion parameters for plates 1 and $2: \delta_{D}^{\text {plates }}=0.3$ and $\delta_{K}^{\text {plates }}=0.15$, and for the complex joint: $\delta_{D}^{C J}=0.8$ and $\delta_{K}^{C J}=0.8$.

The experimental data base is made with 29 accelerometers for 21 experimental configurations corresponding to 21 random distributions of bolt-prestresses having the same mean values. The experimental results are expressed in terms of Shock Response Spectra (SRS) calculated for the acceleration reponses, normalized with respect to $g=9.81 \mathrm{~m} . \mathrm{s}^{-2}$ and denoted by $s(\omega)$ with a fixed damping ratio 0.001 . For a given observation, the results given by the nonparametric probabilistic approach of random uncertainties are displayed by an SRS confidence region described with an upper $\left(S^{+}(\omega)\right)$ and a lower $\left(S^{-}(\omega)\right)$ envelope of the SRS related to a given probability level 
$P c=0.95$. Let $m_{1}(\omega)=E\{s(\omega)\}, m_{2}(\omega)=E\left\{s^{2}(\omega)\right\}$ and $\sigma(\omega)=\sqrt{m_{2}(\omega)-m_{1}^{2}(\omega)}$ be the moments estimated by the usual mathematical statistics. Three curves are defined: (1) $S^{0}(\omega)=10 \log _{10}\left(m_{1}(\omega)\right)$, which is related to the mean function of the stochastic response, (2) $S^{+}(\omega)=10 \log _{10}\left(m_{1}(\omega)+\frac{\sigma(\omega)}{\sqrt{1-P c}}\right)$, which is the the upper envelope of the SRS, and (3) $S^{-}(\omega)=2 S^{0}(\omega)-S^{+}(\omega)$, which is the lower envelope of the SRS. The calculations are carried out using 1000 realizations in the Monte-Carlo numerical method. A convergence analysis with respect to the dimension of the reduced stochastic model of each substructure has been performed. The results presented below correspond to dimensions $n_{1}=50$ for plate $1, n_{2}=67$ for plate 2 and $n_{\mathrm{CJ}}=8$ for the complex joint. For observation 2 located in plate 1 while the excitation is applied in plate 2 (see Figure 9), Figure 11 shows the experimental SRS compared with the confidence region constructed with nonparametric probabilistic approach. The thick dashed line represents the mean model. The solid lines are relative to the 21 experimental configurations. The gray region is the $95 \%$ confidence region of the stochastic response, computed with the nonparametric probabilistic approach of random uncertainties.

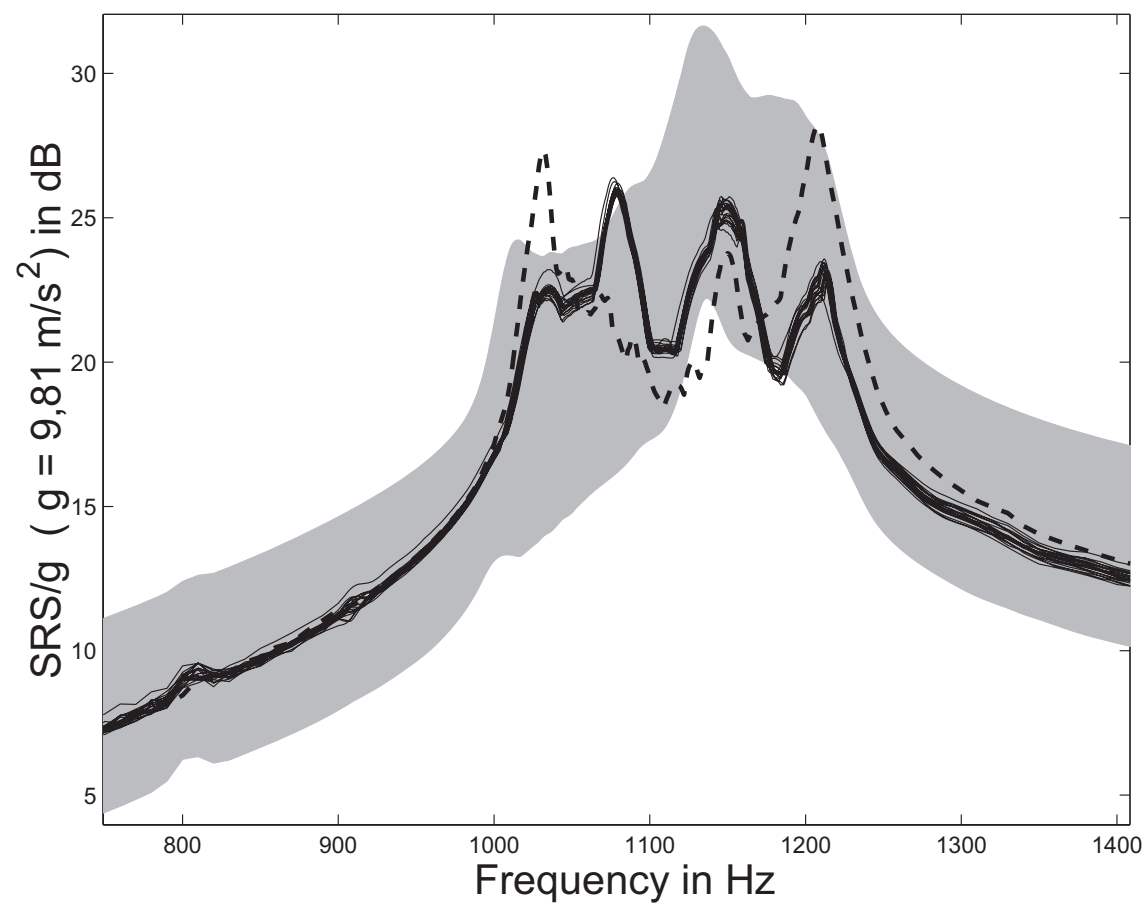

Fig. 11. Shock Response Spectrum at observation point 2 in plate 1 with a point force excitation applied in plate 2.

The comparison of the developed probabilistic model with the experiments shows the capability of the nonparametric probabilistic approach to predict the shock-induced transient response in the 
medium-frequency ranges. In spite of a rather large number of DOFs used in the mean finite element model, significant errors appear between experiment and numerical prediction although the mean finite element model of the complex joint has been updated using experiments (compare the dashed line with the solid lines in Figure 11). These errors are mainly due to the simplified model used for modeling the complex joint, that is to say, are due to model uncertainties mainly located in the complex joint.

\section{Identification of the dispersion parameters of the nonparametric probabilistic approach for linear dynamical systems with data uncertainties and model uncertainties}

In this section, we present a methodology for the experimental identification of the dispersion parameters of the nonparametric probabilistic approach in linear structural dynamics.

\subsection{Experimental modal analysis}

Let us consider $r$ realizations $\mathcal{S}\left(\theta_{1}\right), \ldots, \mathcal{S}\left(\theta_{r}\right)$ of a complex structure $\mathcal{S}$. The modal properties of each realization $\mathcal{S}\left(\theta_{\alpha}\right)$ are experimentally identified by using the usual experimental modal analysis $[7,18]$. Let $m_{\text {in }}$ be the number of measured DOFs and $m_{\text {out }}$ be the number of excited DOFs which are assumed to be the same for all the realizations $\mathcal{S}\left(\theta_{1}\right), \ldots, \mathcal{S}\left(\theta_{r}\right)$. The Frequency Response Function (FRF) $\left[h^{\exp }\left(\omega, \theta_{\alpha}\right)\right]$ of the MIMO system with the $m_{\text {in }}$ inputs and the $m_{\text {out }}$ outputs associated with structure $\mathcal{S}\left(\theta_{\alpha}\right)$ is experimentally identified over the frequency band $\left.\left.B=\right] 0, \omega_{\max }\right]$,

$$
B \longrightarrow M_{\mathrm{m}_{\text {out }}, \mathrm{m}_{\text {in }}}(\mathbb{C}) \quad: \quad \omega \mapsto\left[h^{\exp }\left(\omega, \theta_{\alpha}\right)\right]
$$

From the measured FRF $\omega \mapsto\left[h^{\exp }\left(\omega, \theta_{\alpha}\right)\right]$ identified over frequency band $B$, the experimental modal analysis allows the following information to be constructed:

1. The $n$ first eigenfrequencies $0<\omega_{1}^{\exp }\left(\theta_{\alpha}\right) \leq \ldots \leq \omega_{n}^{\exp }\left(\theta_{\alpha}\right)$ of the elastic modes lying in frequency band $B$.

2. The matrix $\left[\Psi_{n}^{\exp }\left(\theta_{\alpha}\right)\right] \in \mathbb{M}_{\mathrm{m}_{\text {out }}, n}(\mathbb{R})$ of the $n$ elastic modes of vibration associated with the $n$ first eigenfrequencies $\omega_{1}^{\exp }\left(\theta_{\alpha}\right), \ldots, \omega_{n}^{\exp }\left(\theta_{\alpha}\right)$.

3. The diagonal matrix $\left[\widetilde{M}_{n}^{\exp }\left(\theta_{\alpha}\right)\right] \in \mathbb{M}_{n}^{+}(\mathbb{R})$ of the generalized masses such that $\left[\widetilde{M}_{n}^{\exp }\left(\theta_{\alpha}\right)\right]_{j k}=$ $\widetilde{\mu}_{k}^{\exp }\left(\theta_{\alpha}\right) \delta_{j k}$ in which $\widetilde{\mu}_{k}^{\exp }\left(\theta_{\alpha}\right)$ is the generalized mass of elastic mode $k$ and where $\delta_{k k}=1$ and $\delta_{j k}=0$ for $j \neq k$.

C. Soize - Computer Methods in Applied Mechanics and Engineering (CMAME) (accepted in March 2004) 
4. The diagonal matrix $\left[\widetilde{K}_{n}^{\exp }\left(\theta_{\alpha}\right)\right] \in \mathbb{M}_{n}^{+}(\mathbb{R})$ of the generalized stiffnesses such that $\left[\widetilde{K}_{n}^{\exp }\left(\theta_{\alpha}\right)\right]_{j k}=$ $\widetilde{\mu}_{k}^{\exp }\left(\theta_{\alpha}\right) \omega_{k}^{\exp }\left(\theta_{\alpha}\right)^{2} \delta_{j k}$

5. The matrix $\left[\widetilde{D}_{n}^{\exp }\left(\theta_{\alpha}\right)\right] \in \mathbb{M}_{n}^{+}(\mathbb{R})$ of the generalized dampings. In general, this matrix is not diagonal but is full. If the diagonal terms are only experimentally identified, then we have $\left[\widetilde{D}_{n}^{\exp }\left(\theta_{\alpha}\right)\right]_{j k}=2 \widetilde{\xi}_{k}^{\exp }\left(\theta_{\alpha}\right) \widetilde{\mu}_{k}^{\exp }\left(\theta_{\alpha}\right) \omega_{k}^{\exp }\left(\theta_{\alpha}\right) \delta_{j k}$, in which $\widetilde{\xi}_{k}^{\exp }\left(\theta_{\alpha}\right)$ is the modal damping rate of elastic mode $k$.

Finally, from the experimental modal analysis perfomed for each structure $\mathcal{S}\left(\theta_{\alpha}\right)$, it can be deduced the experimental reduced matrices,

$$
\left[\widetilde{M}_{n}^{\exp }\left(\theta_{\alpha}\right)\right] \in \mathbb{M}_{n}^{+}(\mathbb{R}) \quad, \quad\left[\widetilde{D}_{n}^{\exp }\left(\theta_{\alpha}\right)\right] \in \mathbb{M}_{n}^{+}(\mathbb{R}) \quad, \quad\left[\widetilde{K}_{n}^{\exp }\left(\theta_{\alpha}\right)\right] \in \mathbb{M}_{n}^{+}(\mathbb{R})
$$

relative to the $\mathbb{C}^{n}$-valued vector of the experimental generalized coordinates $\widetilde{\mathbf{Q}}^{\exp }\left(\omega, \theta_{\alpha}\right)$ such that

$$
\begin{aligned}
\mathbf{Z}^{\exp }\left(\omega, \theta_{\alpha}\right) & \simeq\left[\Psi_{n}^{\exp }\left(\theta_{\alpha}\right)\right] \widetilde{\mathbf{Q}}^{\exp }\left(\omega, \theta_{\alpha}\right) \\
& =\left[h^{\exp }\left(\omega, \theta_{\alpha}\right)\right] \mathbf{f}^{\exp }(\omega)
\end{aligned}
$$

in which $\mathbf{Z}^{\exp }\left(\omega, \theta_{\alpha}\right) \in \mathbb{C}^{\mathrm{m}_{\text {out }}}$ is the frequency response of the $m_{\text {out }}$ output DOFs induced by the complex vector $\mathbf{f}^{\exp }\left(\theta_{\alpha}\right) \in \mathbb{C}^{m_{\text {in }}}$ of the $m_{\text {in }}$ input DOFs, and where vector $\widetilde{\mathbf{Q}}^{\exp }\left(\omega, \theta_{\alpha}\right)$ has to verify the following matrix equation,

$$
\left(-\omega^{2}\left[\widetilde{M}_{n}^{\exp }\left(\theta_{\alpha}\right)\right]+i \omega\left[\widetilde{D}_{n}^{\exp }\left(\theta_{\alpha}\right)\right]+\left[\widetilde{K}_{n}^{\exp }\left(\theta_{\alpha}\right)\right]\right) \widetilde{\mathbf{Q}}^{\exp }\left(\omega, \theta_{\alpha}\right)=\mathbf{F}^{\exp }\left(\omega, \theta_{\alpha}\right)
$$

with $\mathbf{F}^{\exp }\left(\omega, \theta_{\alpha}\right)=\left[\Psi_{n}^{\exp }\left(\theta_{\alpha}\right)\right]^{T} \mathbf{f}^{\exp }(\omega)$ is the $\mathbb{C}^{n}$-valued vector of the experimental generalized forces.

\subsection{The mean finite element model}

In the frequency domain, the mean reduced model of structure $\mathcal{S}$ constructed from its mean finite element model (see Section 4.2) is written as

$$
\underline{\mathbf{y}}(\omega)=\left[\underline{\Phi}_{n}\right] \underline{\mathbf{q}}(\omega)
$$

in which $\underline{\mathbf{y}}(\omega) \in \mathbb{C}^{m}$ is the complex vector of the $m$ DOFs of the mean finite element model, where $\underline{\mathbf{q}}(\omega) \in \mathbb{C}^{n}$ is the complex vector of the $n$ generalized coordinates of the mean reduced model and where $\left[\underline{\Phi}_{n}\right] \in \mathbb{M}_{m, n}(\mathbb{R})$ is the matrix of the $n$ elastic modes of vibration associated with the $n$ first 
eigenfrequencies $0<\underline{\omega}_{1} \leq \ldots \leq \underline{\omega}_{n}$ and which are computed with the mean finite element model. Vector $\underline{\mathbf{q}}(\omega)$ has to verify the following matrix equation

$$
\left(-\omega^{2}\left[\underline{M}_{n}\right]+i \omega\left[\underline{D}_{n}\right]+\left[\underline{K}_{n}\right]\right) \underline{\mathbf{q}}(\omega)=\mathbf{F}^{n}(\omega) \quad, \quad \forall \omega \in B \quad
$$

in which $\mathbf{F}^{n}(\omega) \in \mathbb{C}^{n}$ is the complex vector of the generalized external forces and where the mean reduced matrices $\left[\underline{M}_{n}\right],\left[\underline{D}_{n}\right]$ and $\left[\underline{K}_{n}\right]$ are such that

$$
\left[\underline{M}_{n}\right] \in \mathbb{M}_{n}^{+}(\mathbb{R}) \quad, \quad\left[\underline{D}_{n}\right] \in \mathbb{M}_{n}^{+}(\mathbb{R}) \quad, \quad\left[\underline{K}_{n}\right] \in \mathbb{M}_{n}^{+}(\mathbb{R})
$$

It should be noted that the number $m$ of DOFs of the mean finite element model is such that $m \gg m_{\text {out }}$ and $m \gg n$.

\subsection{Nonparametric probabilistic model}

The nonparametric probabilistic model of random unceratinties associated with the mean reduced model defined by Eqs. (102) to (104) is constructed as explained in Section 4.3 and is then written as

$$
\begin{gathered}
\mathbf{Y}(\omega)=\left[\underline{\Phi}_{n}\right] \mathbf{Q}(\omega), \\
\left(-\omega^{2}\left[\mathbf{M}_{n}\right]+i \omega\left[\mathbf{D}_{n}\right]+\left[\mathbf{K}_{n}\right]\right) \mathbf{Q}(\omega)=\mathbf{F}^{n}(\omega) \quad, \quad \forall \omega \in B,
\end{gathered}
$$

in which the random reduced matrices with values in $\mathbb{M}_{n}^{+}(\mathbb{R})$ are written as

$$
\left[\mathbf{M}_{n}\right]=\left[\underline{L}_{M_{n}}\right]^{T}\left[\mathbf{G}_{M_{n}}\right]\left[\underline{L}_{M_{n}}\right],\left[\mathbf{D}_{n}\right]=\left[\underline{L}_{D_{n}}\right]^{T}\left[\mathbf{G}_{D_{n}}\right]\left[\underline{L}_{D_{n}}\right],\left[\mathbf{K}_{n}\right]=\left[\underline{L}_{K_{n}}\right]^{T}\left[\mathbf{G}_{K_{n}}\right]\left[\underline{L}_{K_{n}}\right],
$$

where random matrices $\left[\mathbf{G}_{M_{n}}\right],\left[\mathbf{G}_{D_{n}}\right]$ and $\left[\mathbf{G}_{K_{n}}\right]$ are in ensemble $\mathrm{SG}^{+}$defined in Section 2.1 and where the upper triangular matrices $\left[\underline{L}_{M_{n}}\right],\left[\underline{L}_{D_{n}}\right]$ and $\left[\underline{L}_{K_{n}}\right]$ in $M_{n}(\mathbb{R})$ are such

$$
\left[\underline{M}_{n}\right]=\left[\underline{L}_{M_{n}}\right]^{T}\left[\underline{L}_{M_{n}}\right] \quad, \quad\left[\underline{D}_{n}\right]=\left[\underline{L}_{D_{n}}\right]^{T}\left[\underline{L}_{D_{n}}\right] \quad, \quad\left[\underline{K}_{n}\right]=\left[\underline{L}_{K_{n}}\right]^{T}\left[\underline{L}_{K_{n}}\right] \quad .
$$

The probability distribution of each random matrix $\left[\mathbf{G}_{M_{n}}\right],\left[\mathbf{G}_{D_{n}}\right]$ or $\left[\mathbf{G}_{K_{n}}\right]$ is defined in Section 2.1.3 and its algebraic representation is defined in Section 2.1.7. These probability distributions depends on the dispersion parameters $\delta_{M}, \delta_{D}$ and $\delta_{K}$ which are defined by Eq. (37) and which are the parameters which have to be identified using the experimental data. 


\subsection{Transformation of the experimental reduced matrices}

In order to identify the dispersion parameters $\delta_{M}, \delta_{D}$ and $\delta_{K}$ of the nonparametric probabilistic model using experimental data, we have to construct experimental realizations of random reduced matrices $\left[\mathbf{M}_{n}\right],\left[\mathbf{D}_{n}\right]$ and $\left[\mathbf{K}_{n}\right]$. It should be noted that experimental realizations $\left[\widetilde{M}_{n}^{\exp }\left(\theta_{\alpha}\right)\right]$, $\left[\widetilde{D}_{n}^{\exp }\left(\theta_{\alpha}\right)\right]$ and $\left[\widetilde{K}_{n}^{\exp }\left(\theta_{\alpha}\right)\right]$ cannot directly be used as realizations of random reduced matrices $\left[\mathbf{M}_{n}\right]$, $\left[\mathbf{D}_{n}\right]$ and $\left[\mathbf{K}_{n}\right]$ because these two sets of matrices do not correspond to the same basis (i.e. to the same generalized coordinates). We then have to transform the experimental reduced matrices utilizing an adapted change of basis in order to get experimental realizations of the random reduced matrices.

The $\mathbb{C}^{m}$-valued random vector $\mathbf{Y}(\omega)$ of the DOFs of the stochastic dynamical system is defined by Eq. (105). If all these $m$ DOFs were measured, then the $\mathbb{C}^{m}$-valued vector $\mathbf{Y}^{\exp }\left(\omega, \theta_{\alpha}\right)$ could be measured for each realization $\mathcal{S}\left(\theta_{\alpha}\right)$ of structure $\mathcal{S}$. In point of fact, only a small number of

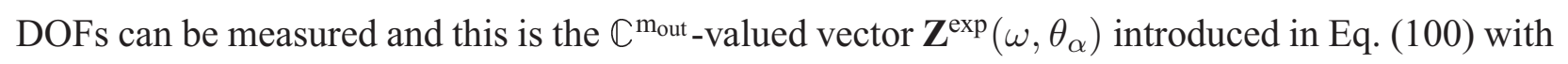
$m \gg m_{\text {out }}$. Consequently, there is a matrix $[P] \in \mathbb{M}_{\mathrm{m}_{\text {out }}, m}(\mathbb{R})$ constituted of 0 and 1 such that

$$
\mathbf{Z}^{\exp }\left(\omega, \theta_{\alpha}\right)=[P] \mathbf{Y}^{\exp }\left(\omega, \theta_{\alpha}\right)
$$

Taken into account Eq. (105), the vector of the generalized coordinates associated with $\mathbf{Y}^{\exp }\left(\omega, \theta_{\alpha}\right)$ is then $\mathbf{Q}^{\exp }\left(\omega, \theta_{\alpha}\right) \in \mathbb{C}^{n}$ such that

$$
\mathbf{Y}^{\exp }\left(\omega, \theta_{\alpha}\right)=\left[\underline{\Phi}_{n}\right] \mathbf{Q}^{\exp }\left(\omega, \theta_{\alpha}\right)
$$

Substituting Eq. (110) into Eq. (109) yields

$$
\mathbf{Z}^{\exp }\left(\omega, \theta_{\alpha}\right)=[P]\left[\underline{\Phi}_{n}\right] \mathbf{Q}^{\exp }\left(\omega, \theta_{\alpha}\right)
$$

From Eqs. (100) and (111), we deduced that

$$
\left[\Psi_{n}^{\exp }\left(\theta_{\alpha}\right)\right] \widetilde{\mathbf{Q}}^{\exp }\left(\omega, \theta_{\alpha}\right)=[P]\left[\underline{\Phi}_{n}\right] \mathbf{Q}^{\exp }\left(\omega, \theta_{\alpha}\right)
$$

By construction, the matrix $\left[\Psi_{n}^{\exp }\left(\theta_{\alpha}\right)\right]^{T}\left[\Psi_{n}^{\exp }\left(\theta_{\alpha}\right)\right] \in \mathbb{M}_{n}(\mathbb{R})$ is invertible. Introducing the left pseudo-inverse $\left(\left[\Psi_{n}^{\exp }\left(\theta_{\alpha}\right)\right]^{T}\left[\Psi_{n}^{\exp }\left(\theta_{\alpha}\right)\right]\right)^{-1}\left[\Psi_{n}^{\exp }\left(\theta_{\alpha}\right)\right]^{T} \in \mathbb{M}_{n}, \mathrm{~m}_{\text {out }}(\mathbb{R})$ of $\left[\Psi_{n}^{\exp }\left(\theta_{\alpha}\right)\right] \in \mathbb{M}_{\mathrm{m}_{\text {out }}, n}(\mathbb{R})$, Eq. (112) yields

$$
\widetilde{\mathbf{Q}}^{\exp }\left(\omega, \theta_{\alpha}\right)=\left[S_{n}^{\exp }\left(\theta_{\alpha}\right)\right] \mathbf{Q}^{\exp }\left(\omega, \theta_{\alpha}\right)
$$


in which the matrix $\left[S_{n}^{\exp }\left(\theta_{\alpha}\right)\right] \in \mathbb{M}_{n}(\mathbb{R})$ is given by

$$
\left[S_{n}^{\exp }\left(\theta_{\alpha}\right)\right]=\left(\left[\Psi_{n}^{\exp }\left(\theta_{\alpha}\right)\right]^{T}\left[\Psi_{n}^{\exp }\left(\theta_{\alpha}\right)\right]\right)^{-1}\left[\Psi_{n}^{\exp }\left(\theta_{\alpha}\right)\right]^{T}[P]\left[\underline{\Phi}_{n}\right]
$$

Substituting Eq. (113) into Eq. (101) yields

$$
\begin{aligned}
\left(-\omega^{2}\left[\widetilde{M}_{n}^{\exp }\left(\theta_{\alpha}\right)\right]\left[S_{n}^{\exp }\left(\theta_{\alpha}\right)\right]+i \omega\left[\widetilde{D}_{n}^{\exp }\left(\theta_{\alpha}\right)\right]\left[S_{n}^{\exp }\left(\theta_{\alpha}\right)\right]\right. & \left.+\left[\widetilde{K}_{n}^{\exp }\left(\theta_{\alpha}\right)\right]\left[S_{n}^{\exp }\left(\theta_{\alpha}\right)\right]\right) \mathbf{Q}^{\exp }\left(\omega, \theta_{\alpha}\right) \\
& =\mathbf{F}^{\exp }\left(\omega, \theta_{\alpha}\right)
\end{aligned}
$$

The left multiplication of Eq. (115) by $\left[S_{n}^{\exp }\left(\theta_{\alpha}\right)\right]^{T}$ yields

$$
\left(-\omega^{2}\left[M_{n}^{\exp }\left(\theta_{\alpha}\right)\right]+i \omega\left[D_{n}^{\exp }\left(\theta_{\alpha}\right)\right]+\left[K_{n}^{\exp }\left(\theta_{\alpha}\right)\right]\right) \mathbf{Q}^{\exp }\left(\omega, \theta_{\alpha}\right)=\left[S_{n}^{\exp }\left(\theta_{\alpha}\right)\right]^{T} \mathbf{F}^{\exp }\left(\omega, \theta_{\alpha}\right)
$$

in which the matrices $\left[M_{n}^{\exp }\left(\theta_{\alpha}\right)\right],\left[D_{n}^{\exp }\left(\theta_{\alpha}\right)\right]$ and $\left[K_{n}^{\exp }\left(\theta_{\alpha}\right)\right]$ are defined by

$$
\begin{aligned}
& {\left[M_{n}^{\exp }\left(\theta_{\alpha}\right)\right]=\left[S_{n}^{\exp }\left(\theta_{\alpha}\right)\right]^{T}\left[\widetilde{M}_{n}^{\exp }\left(\theta_{\alpha}\right)\right]\left[S_{n}^{\exp }\left(\theta_{\alpha}\right)\right] \in \mathbb{M}_{n}^{+}(\mathbb{R})} \\
& {\left[D_{n}^{\exp }\left(\theta_{\alpha}\right)\right]=\left[S_{n}^{\exp }\left(\theta_{\alpha}\right)\right]^{T}\left[\widetilde{D}_{n}^{\exp }\left(\theta_{\alpha}\right)\right]\left[S_{n}^{\exp }\left(\theta_{\alpha}\right)\right] \in \mathbb{M}_{n}^{+}(\mathbb{R})} \\
& {\left[K_{n}^{\exp }\left(\theta_{\alpha}\right)\right]=\left[S_{n}^{\exp }\left(\theta_{\alpha}\right)\right]^{T}\left[\widetilde{K}_{n}^{\exp }\left(\theta_{\alpha}\right)\right]\left[S_{n}^{\exp }\left(\theta_{\alpha}\right)\right] \in \mathbb{M}_{n}^{+}(\mathbb{R})}
\end{aligned}
$$

Comparing Eqs.(105)-(106) with Eqs. (110)-(116), we deduce that matrices $\left[M_{n}^{\exp }\left(\theta_{\alpha}\right)\right],\left[D_{n}^{\exp }\left(\theta_{\alpha}\right)\right]$ and $\left[K_{n}^{\exp }\left(\theta_{\alpha}\right)\right]$ defined by Eq. (117) can be considered as experimental realizations of random matrices $\left[\mathbf{M}_{n}\right],\left[\mathbf{D}_{n}\right]$ and $\left[\mathbf{K}_{n}\right]$ defined by Eq. (107).

\subsection{Experimental identification of the dispersion parameters of the nonparametric probabilistic} model

The problem solved in this section is the experimental identification of the dispersion parameters $\delta_{M}, \delta_{D}$ and $\delta_{K}$ of the nonparametric probabilistic model using experimental data and two types of estimators: (1) the natural estimator deduced from Eq. (37) and (2) the estimator constructed with the maximum likelihood method. Let $\left[A_{n}^{\exp }\left(\theta_{\alpha}\right)\right]$ representing $\left[M_{n}^{\exp }\left(\theta_{\alpha}\right)\right],\left[D_{n}^{\exp }\left(\theta_{\alpha}\right)\right]$ or $\left[K_{n}^{\exp }\left(\theta_{\alpha}\right)\right]$, let $\left[\mathbf{A}_{n}\right]$ representing $\left[\mathbf{M}_{n}\right],\left[\mathbf{D}_{n}\right]$ and $\left[\mathbf{K}_{n}\right]$ and let $\delta_{A}$ representing $\delta_{M}, \delta_{D}$ and $\delta_{K}$. Consequently, we have to identify dispersion parameter $\delta_{A}$ of random matrix $\left[\mathbf{A}_{n}\right]$ knowing $r$ experimental realizations $\left[A_{n}^{\exp }\left(\theta_{1}\right)\right], \ldots,\left[A_{n}^{\exp }\left(\theta_{r}\right)\right]$ of random matrix $\left[\mathbf{A}_{n}\right]$. 


\subsubsection{Experimental realizations of the normalized factorized random matrix}

Since $\left[\mathbf{A}_{n}\right]$ belongs to ensemble $\mathrm{SE}^{+}$, from Section 2.2, we deduced that random matrix $\left[\mathbf{A}_{n}\right]$ with values in $\mathbb{M}_{n}^{+}(\mathbb{R})$ is written as $\left[\mathbf{A}_{n}\right]=\left[\underline{L}_{A_{n}}\right]^{T}\left[\mathbf{G}_{n}\right]\left[\underline{L}_{A_{n}}\right]$ in which the invertible upper triangular matrix $\left[\underline{L}_{A_{n}}\right] \in \mathbb{M}_{n}(\mathbb{R})$ is such that $\left[\underline{A}_{n}\right]=\left[\underline{L}_{A_{n}}\right]^{T}\left[\underline{L}_{A_{n}}\right] \in \mathbb{M}_{n}^{+}(\mathbb{R})$ and where random matrix $\left[\mathbf{G}_{n}\right]$ belonging to ensemble $\mathrm{SG}^{+}$is written (see Eq. (23)) as $\left[\mathbf{G}_{n}\right]=\left[\mathbf{L}_{n}\right]^{T}\left[\mathbf{L}_{n}\right]$ with $\left[\mathbf{L}_{n}\right]$ the random matrix defined in Section 2.1.7. The objective of this section is to construct $r$ experimental realizations $\left[L_{n}^{\exp }\left(\theta_{1}\right)\right], \ldots,\left[L_{n}^{\exp }\left(\theta_{r}\right)\right]$ of random matrix $\left[\mathbf{L}_{n}\right]$. We then introduce the matrix $\left[G_{n}^{\exp }\left(\theta_{\alpha}\right)\right] \in \mathbb{M}_{n}^{+}(\mathbb{R})$ such that $\left[A_{n}^{\exp }\left(\theta_{\alpha}\right)\right]=\left[\underline{L}_{A_{n}}\right]^{T}\left[G_{n}^{\exp }\left(\theta_{\alpha}\right)\right]\left[\underline{L}_{A_{n}}\right]$. Since matrix $\left[\underline{L}_{A_{n}}\right]$ is invertible, matrix $\left[G_{n}^{\exp }\left(\theta_{\alpha}\right)\right]$ is then given by the equation,

$$
\left[G_{n}^{\exp }\left(\theta_{\alpha}\right)\right]=\left[\underline{L}_{A_{n}}\right]^{-T}\left[A_{n}^{\exp }\left(\theta_{\alpha}\right)\right]\left[\underline{L}_{A_{n}}\right]^{-1} \in \mathbb{M}_{n}^{+}(\mathbb{R})
$$

Matrix $\left[G_{n}^{\exp }\left(\theta_{\alpha}\right)\right]$ can then be written as

$$
\left[G_{n}^{\exp }\left(\theta_{\alpha}\right]=\left[L_{n}^{\exp }\left(\theta_{\alpha}\right)\right]^{T}\left[L_{n}^{\exp }\left(\theta_{\alpha}\right)\right]\right.
$$

in which the upper triangular matrix $\left[L_{A_{n}}^{\exp }\left(\theta_{\alpha}\right)\right]$ is calculated by the Cholesky factorization of positive-definite matrix $\left[G_{n}^{\exp }\left(\theta_{\alpha}\right)\right]$. Consequently, we have effectively constructed $r$ realizations $\left[L_{n}^{\exp }\left(\theta_{1}\right)\right], \ldots,\left[L_{n}^{\exp }\left(\theta_{r}\right)\right]$ of random matrix $\left[\mathbf{L}_{n}\right]$.

\subsubsection{Probability density function of random matrix $\left[\mathbf{L}_{n}\right]$}

Let $\sigma_{A}$ be the parameter defined in Section 2.1.7 such that

$$
\sigma_{A}=\frac{\delta_{A}}{\sqrt{n+1}}
$$

Therefore, it is equivalent to identified $\sigma_{A}$ or $\delta_{A}$. From Section 2.1.7 and since the random variables $\left\{\left[\mathbf{L}_{n}\right]_{j j^{\prime}}, j \leq j^{\prime}\right\}$ are independent, the probability density function $p_{\left[\mathbf{L}_{n}\right]}\left(\left[L_{n}\right] ; \sigma_{A}\right)$ of random upper triangular matrix $\left[\mathbf{L}_{n}\right]$, with respect to the measure $d L_{n}=\Pi_{1 \leq j \leq j^{\prime} \leq n} d\left[L_{n}\right]_{j j^{\prime}}$, is written as

$$
p_{\left[\mathbf{L}_{n}\right]}\left(\left[L_{n}\right] ; \sigma_{A}\right)=\left\{\Pi_{1 \leq j<j^{\prime} \leq n} p_{j j^{\prime}}\left(\left[L_{n}\right]_{j j^{\prime}} ; \sigma_{A}\right)\right\}\left\{\Pi_{1 \leq j \leq n} p_{j}\left(\left[L_{n}\right]_{j j} ; \sigma_{A}\right)\right\}
$$

in which

$$
\begin{aligned}
p_{j j^{\prime}}\left(\left[L_{n}\right]_{j j^{\prime}} ; \sigma_{A}\right) & =\frac{1}{\sqrt{2 \pi} \sigma_{A}} \exp \left\{-\frac{1}{2 \sigma_{A}^{2}}\left[L_{n}\right]_{j j^{\prime}}^{2}\right\}, \\
p_{j}\left(\left[L_{n}\right]_{j j} ; \sigma_{A}\right) & =\mathbb{1}_{\mathbb{R}^{+}}\left(\left[L_{n}\right]_{j j}\right) \times \frac{2}{\Gamma\left(\frac{1}{2 \sigma_{A}^{2}}+\frac{1-j}{2}\right)} \\
& \times\left(2 \sigma_{A}^{2}\right)^{-1 /\left(2 \sigma_{A}^{2}\right)-(1-j) / 2}\left[L_{n}\right]_{j j}^{\sigma_{A}^{-2}-j} \exp \left\{-\frac{1}{2 \sigma_{A}^{2}}\left[L_{n}\right]_{j j}^{2}\right\} .
\end{aligned}
$$




\subsubsection{Independent copies of random matrix $\left[\mathbf{L}_{n}^{\exp }\right]$}

Let $\left[\mathbf{L}_{n}^{\exp , 1}\right], \ldots,\left[\mathbf{L}_{n}^{\exp , r}\right]$ be $r$ independent copies of random matrix $\left[\mathbf{L}_{n}^{\exp }\right]$. A realization $\left[\mathbf{L}_{n}^{\exp , 1}(\theta)\right]$, $\ldots,\left[\mathbf{L}_{n}^{\exp , r}(\theta)\right]$ of the $r$ random matrices $\left[\mathbf{L}_{n}^{\exp , 1}\right], \ldots,\left[\mathbf{L}_{n}^{\exp , r}\right]$ can be constructed in using the $r$ known independent realizations $\left[L_{n}^{\exp }\left(\theta_{1}\right)\right], \ldots,\left[L_{n}^{\exp }\left(\theta_{r}\right)\right]$ of random matrix $\left[\mathbf{L}_{n}\right]$ whose probability density function $p_{\left[\mathbf{L}_{n}\right]}\left(\left[L_{n}\right] ; \sigma_{A}\right)$ is defined by Eq. (121). For $\alpha=1, \ldots, r$, we then take

$$
\left[\mathbf{L}_{n}^{\exp , \alpha}(\theta)\right]=\left[L_{n}^{\exp }\left(\theta_{\alpha}\right)\right]
$$

\subsubsection{Estimation of $\delta_{A}$ by using the natural method}

From Eq. (37), the natural estimator $\widehat{\delta}_{r}^{0}$ of $\delta_{A}$ is the random variable $\widehat{\delta}_{r}^{0}=d_{r}\left(\left[\mathbf{L}_{n}^{\exp , 1}\right], \ldots,\left[\mathbf{L}_{n}^{\exp , r}\right]\right)$ which is defined by

$$
\begin{aligned}
\widehat{\delta}_{r}^{0} & =d_{r}\left(\left[\mathbf{L}_{n}^{\exp , 1}\right], \ldots,\left[\mathbf{L}_{n}^{\exp , r}\right]\right) \\
& =\left\{\frac{1}{n r} \sum_{\alpha=1}^{r}\left\|\left[\mathbf{L}_{n}^{\exp , \alpha}\right]^{T}\left[\mathbf{L}_{n}^{\exp , \alpha}\right]-\left[I_{n}\right]\right\|_{F}^{2}\right\}^{1 / 2} .
\end{aligned}
$$

The mean value $m_{\widehat{\delta}_{r}^{0}}$ and the standard deviation $\sigma_{\widehat{\delta}_{r}^{0}}$ of estimator $\widehat{\delta}_{r}^{0}$ are usually defined by

$$
m_{\widehat{\delta}_{r}^{0}}=E\left\{\widehat{\delta}_{r}^{0}\right\} \quad, \quad \sigma_{\widehat{\delta}_{r}^{0}}^{2}=E\left\{\left(\widehat{\delta}_{r}^{0}-m_{\widehat{\delta}_{r}^{0}}\right)^{2}\right\} \quad .
$$

The estimation of $\delta_{A}$ is given as the realization $\widehat{\delta}_{r}^{0}(\theta)=d_{r}\left(\left[\mathbf{L}_{n}^{\exp , 1}(\theta)\right], \ldots,\left[\mathbf{L}_{n}^{\exp , r}(\theta)\right]\right)$ of random variable $\widehat{\delta}_{r}^{0}$ and can then be written as

$$
\widehat{\delta}_{r}^{0}(\theta)=d_{r}\left(\left[L_{n}^{\exp }\left(\theta_{1}\right)\right], \ldots,\left[L_{n}^{\exp }\left(\theta_{r}\right)\right]\right)
$$

\subsubsection{Estimation of $\delta_{A}$ by the maximum likelihood method}

From Eq. (120), we deduce that it is equivalent to estimate $\delta_{A}$ or $\sigma_{A}$. The unknown parameter which has to be estimated can then be chosen as $\sigma_{A}$. The likelihood function is the random variable $\mathcal{L}_{r}\left(\sigma_{A} ;\left[\mathbf{L}_{n}^{\exp , 1}\right], \ldots,\left[\mathbf{L}_{n}^{\exp , r}\right]\right)$ depending on parameter $\sigma_{A}$ such that

$$
\mathcal{L}_{r}\left(\sigma_{A} ;\left[\mathbf{L}_{n}^{\exp , 1}\right], \ldots,\left[\mathbf{L}_{n}^{\exp , r}\right]\right)=\Pi_{\alpha=1}^{r} p_{\left[\mathbf{L}_{n}\right]}\left(\left[\mathbf{L}_{n}^{\exp , \alpha}\right] ; \sigma_{A}\right)
$$

From Eqs (121) to (123), we deduce that

$$
\log \left\{\mathcal{L}_{r}\left(\sigma_{A} ;\left[\mathbf{L}_{n}^{\exp , 1}\right], \ldots,\left[\mathbf{L}_{n}^{\exp , r}\right]\right)\right\}=\sum_{\alpha=1}^{r} \log \left\{p_{\left[\mathbf{L}_{n}\right]}\left(\left[\mathbf{L}_{n}^{\exp , \alpha}\right] ; \sigma_{A}\right)\right\}
$$


in which

$$
\begin{aligned}
\log \left\{p_{\left[\mathbf{L}_{n}\right]}\left(\left[\mathbf{L}_{n}^{\exp , \alpha}\right] ; \sigma_{A}\right)\right\} & =\sum_{1 \leq j<j^{\prime} \leq n} \log \left\{p_{j j^{\prime}}\left(\left[\mathbf{L}_{n}^{\exp , \alpha}\right]_{j j^{\prime}} ; \sigma_{A}\right)\right\} \\
& +\sum_{1 \leq j \leq n} \log \left\{p_{j}\left(\left[\mathbf{L}_{n}^{\exp , \alpha}\right]_{j j} ; \sigma_{A}\right)\right\}
\end{aligned}
$$

with

$$
\begin{gathered}
\log \left\{p_{j j^{\prime}}\left(\left[\mathbf{L}_{n}^{\exp , \alpha}\right]_{j j^{\prime}} ; \sigma_{A}\right)\right\}=-\frac{1}{2 \sigma_{A}^{2}}\left[\mathbf{L}_{n}^{\exp , \alpha}\right]_{j j^{\prime}}^{2}-\log \left\{\sqrt{2 \pi} \sigma_{A}\right\} \\
\log \left\{p_{j}\left(\left[\mathbf{L}_{n}^{\exp , \alpha}\right]_{j j} ; \sigma_{A}\right)\right\}=\log \{2\}-\log \left\{\Gamma\left(\frac{1}{2 \sigma_{A}^{2}}+\frac{1-j}{2}\right)\right\}-\left(\frac{1}{2 \sigma_{A}^{2}}+\frac{1-j}{2}\right) \log \left\{2 \sigma_{A}^{2}\right\} \\
+\left(\frac{1}{\sigma_{A}^{2}}-j\right) \log \left\{\left[\mathbf{L}_{n}^{\exp , \alpha}\right]_{j j}\right\}-\frac{1}{2 \sigma_{A}^{2}}\left[\mathbf{L}_{n}^{\exp , \alpha}\right]_{j j}^{2}
\end{gathered}
$$

The estimator of parameter $\sigma_{A}$ defined by the maximum likelihood method [21] is the random variable $\widehat{\sigma}_{r}=s_{r}\left(\left[\mathbf{L}_{n}^{\exp , 1}\right], \ldots,\left[\mathbf{L}_{n}^{\exp , r}\right]\right)$ which is such that

$$
\mathcal{L}_{r}\left(\widehat{\sigma}_{r} ;\left[\mathbf{L}_{n}^{\exp , 1}\right], \ldots,\left[\mathbf{L}_{n}^{\exp , r}\right]\right)=\max _{\sigma_{A}} \mathcal{L}_{r}\left(\sigma_{A} ;\left[\mathbf{L}_{n}^{\exp , 1}\right], \ldots,\left[\mathbf{L}_{n}^{\exp , r}\right]\right),
$$

or equivalently, by

$$
\log \left\{\mathcal{L}_{r}\left(\widehat{\sigma}_{r}\right) ;\left[\mathbf{L}_{n}^{\exp , 1}\right], \ldots,\left[\mathbf{L}_{n}^{\exp , r}\right]\right\}=\max _{\sigma_{A}} \log \left\{\mathcal{L}_{r}\left(\sigma_{A} ;\left[\mathbf{L}_{n}^{\exp , 1}\right], \ldots,\left[\mathbf{L}_{n}^{\exp , r}\right]\right)\right\} \quad .
$$

The mean value $m_{\widehat{\sigma}_{r}}$ and the standard deviation $\sigma_{\widehat{\sigma}_{r}}$ of estimator $\widehat{\sigma}_{r}$ are usually defined by

$$
m_{\widehat{\sigma}_{r}}=E\left\{\widehat{\sigma}_{r}\right\} \quad, \quad \sigma_{\widehat{\sigma}_{r}}^{2}=E\left\{\left(\widehat{\sigma}_{r}-m_{\widehat{\sigma}_{r}}\right)^{2}\right\} .
$$

Let $m_{\widehat{\delta}_{r}}$ and $\sigma_{\widehat{\delta}_{r}}$ be the mean and the standard deviation of the estimator $\widehat{\delta}_{r}$ of $\delta_{A}$ which is such that $\widehat{\delta}_{r}=\widehat{\sigma}_{r} \sqrt{n+1}$. We then have

$$
m_{\widehat{\delta}_{r}}=m_{\widehat{\sigma}_{r}} \sqrt{n+1} \quad, \quad \sigma_{\widehat{\delta}_{r}}=\sigma_{\widehat{\sigma}_{r}} \sqrt{n+1} .
$$

The estimation of $\sigma_{A}$ is given as the realization $\widehat{\sigma}_{r}(\theta)=s_{r}\left(\left[\mathbf{L}_{n}^{\exp , 1}(\theta)\right], \ldots,\left[\mathbf{L}_{n}^{\exp , r}(\theta)\right]\right)$ of random variable $\widehat{\sigma}_{r}$ and can then be written as

$$
\widehat{\sigma}_{r}(\theta)=s_{r}\left(\left[L_{n}^{\exp }\left(\theta_{1}\right)\right], \ldots,\left[L_{n}^{\exp }\left(\theta_{r}\right)\right]\right) .
$$

Then, the estimation of $\delta_{A}$ is given by $\widehat{\sigma}_{r}(\theta) \sqrt{n+1}$.

For a given realization $\left[L_{n}^{\exp }\left(\theta_{1}\right)\right], \ldots,\left[L_{n}^{\exp }\left(\theta_{r}\right)\right]$, the maximum of the function

$$
\sigma_{A} \mapsto \log \left\{\mathcal{L}_{r}\left(\sigma_{A} ;\left[L_{n}^{\exp }\left(\theta_{1}\right)\right], \ldots,\left[L_{n}^{\exp }\left(\theta_{r}\right)\right]\right)\right\}
$$

is obtained for $\sigma_{A}=\widehat{\sigma}_{r}(\theta)$ and is directly determined in calculating the graph of this function in the neighborhood of $\sigma_{A}^{0}=\widehat{\delta}_{r}^{0}(\theta) / \sqrt{n+1}$ in which $\widehat{\delta}_{r}^{0}(\theta)$ is defined by Eq. (127) and which constitutes a reasonable estimation of $\delta_{A}$. 


\subsubsection{Efficiency and convergence of the two estimators}

The biais and the efficiency of the estimators $\widehat{\delta}_{r}^{0}$ and $\widehat{\delta}_{r}$ of parameter $\delta_{A}$, respectively defined in Sections 5.5.4 and 5.5.5, can be analyzed (1) in comparing the functions $r \mapsto \delta_{A}, r \mapsto m_{\widehat{\delta}_{r}^{0}}$ and $r \mapsto m_{\widehat{\delta}_{r}}$ and (2) in comparing the functions $r \mapsto \sigma_{\widehat{\delta}_{r}^{0}}$ and $r \mapsto \sigma_{\widehat{\delta}_{r}}$, defined by Eqs. (126) and (136). For $n=10$ and $\delta_{A}=0.5$, these functions have been calculated on $r \in[1,20]$, in using the Monte Carlo simulation with 5000 realizations. Figure 12 displays the functions $r \mapsto \delta_{A}, r \mapsto m_{\widehat{\delta}_{r}^{0}}$ and $r \mapsto m_{\widehat{\delta}_{r}}$. It can be seen that, for any $r$ fixed in $[1,20]$, the biais of estimator $\widehat{\delta}_{r}$ is less than the biais of estimator $\widehat{\delta}_{r}^{0}$. Figure 13 displays the functions $r \mapsto \sigma_{\widehat{\delta}_{r}^{0}}$ and $r \mapsto \sigma_{\widehat{\delta}_{r}}$. It can be seen that, for any $r$ fixed in $[1,20]$, estimator $\widehat{\delta}_{r}$ is more efficient than estimator $\widehat{\delta}_{r}^{0}$.

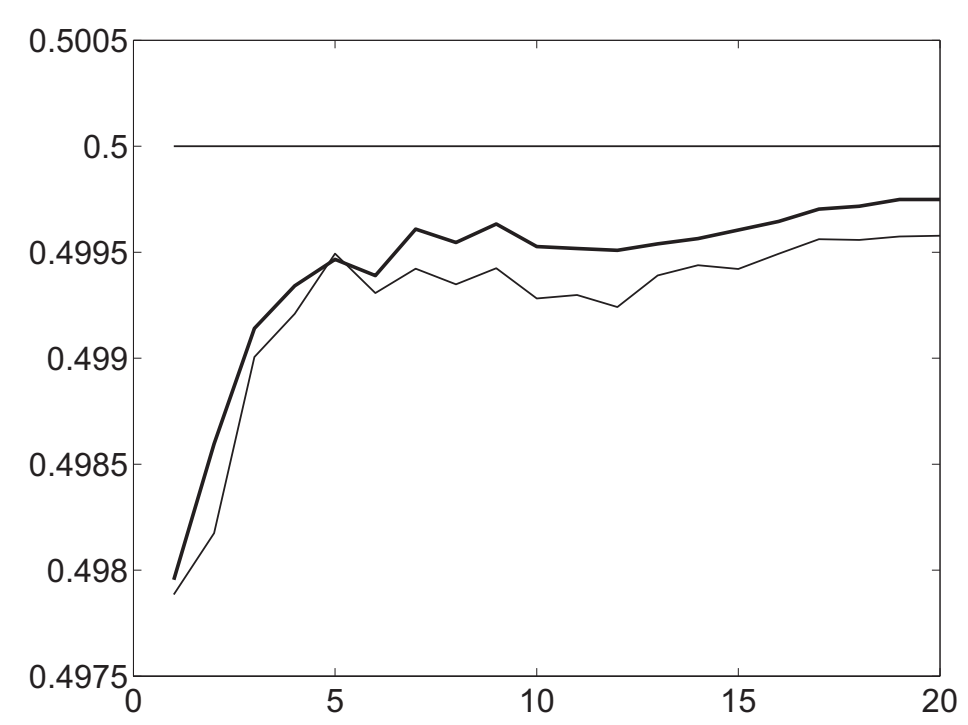

Fig. 12. Graphs of functions $r \mapsto \delta_{A}$ (horizontal thin solid line), $r \mapsto m_{\widehat{\delta_{r}^{0}}}$ (thin solid line) and $r \mapsto m_{\delta_{r}}$ (thick solid line).

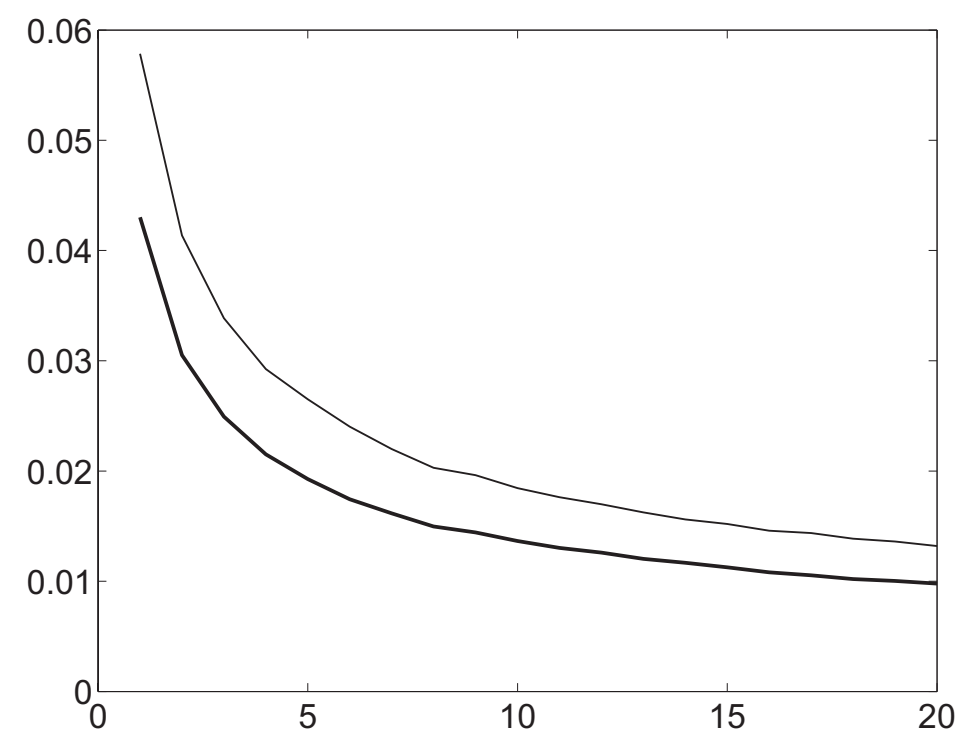

Fig. 13. Graphs of functions $r \mapsto \sigma_{\widehat{\delta}_{r}^{0}}$ (thin solid line) and $r \mapsto \sigma_{\widehat{\delta}_{r}}$ (thick solid line). 


\section{Conclusions}

This paper proposes a nonparametric probabilistic approach for taking into account data and model uncertainties in linear dynamical systems for which the mean model is given and fixed. The objective of such a nonparametric probabilistic approach is to increase the predictability of a given and fixed mean model, that is to say, is to increase the predictability without improving the mean model by introducing additional smaller spatial scales or a hierarchy of models. In such a nonparametric probabilistic approach, data and model uncertainties can be introduced by using the random matrix theory. In this context, several useful sets of random matrices have been introduced and allow uncertainties to be modeled in computational mechanics. Non homogeneous uncertainties can be modeled with such a nonparametric approach using dynamic substructuring methods. For this nonparametric approach, the information used does not require the description of the local parameters of the mathematical-mechanical model. The parametric approaches existing in literature are very useful when the number of uncertain parameters is not too large and when the probabilistic model can be constructed for the set of parameters considered. The nonparametric approach proposed is useful when the number of uncertain parameters is high and/or when the probabilistic model is difficult to construct for the set of parameters considered. In addition, the parametric approaches do not allow the model uncertainties to be taken into account (because a parametric approach is associated with a fixed model exhibiting some parameters), whereas the nonparametric approach proposed allows the model uncertainties to be taken into account for a given and fixed mean model. For some nonlinear dynamical systems constituted of a linear system with additional localized nonlinearities, the nonparametric model is only applied to the linear part of the reduced model. This nonparametric model of random uncertainties can simultaneously be used with the usual parametric model of random uncertainties which allows random uncertainties on nonlinear damping and restoring forces to be taken into account. In this case, a nonparametricparametric model of random uncertainties has to be considered. Concerning this nonparametric model, the probability distribution of each random generalized matrix of the linear part of the random reduced model depends only on two parameters: the mean generalized matrix associated with the mean finite element model and corresponding to the design model, and a scalar parameter $\delta$ whose value has to be fixed by the designer in the interval $[0,1[$ in order to give the dispersion level attached to the random generalized matrix. It seems clear that parameter $\delta$ is a global parameter resulting from expertise or identification. Concerning the identification of parameter $\delta_{A}$ knowing 
experimental data, a methodology and two estimators are proposed. The first one is very easy to construct and the second one, which is based on the maximum likelihood method, is more efficient.

\section{References}

[1] T.W. Anderson, Introduction to Multivariate Statistical Analysis, Wiley, New York, 1958.

[2] I. Babuska, P. Chatzipantelidis, On solving elliptic stochastic partial differential equations, Computer Methods in Applied Mechanics and Engineering 191 (2002) 4093-4122.

[3] H. Chebli, C. Soize, Experimental validation of a nonparametric probabilistic model of non homogeneous uncertainties for dynamical systems, Journal of the Acoustical Society of America 115(2) (2004) 697-705.

[4] R.R. Craig, M.M. Bampton, Coupling of substructures for dynamical analysis, AIAA Journal 6 (1968) 1313-1319.

[5] C. Desceliers, C. Soize and S. Cambier, Nonparametric-parametric model for random uncertainties in nonlinear structural dynamics - Application to earthquake engineering, Earthquake Engineering and Structural Dynamics 33(3) (2004) 315-327 .

[6] J. Duchereau and C. Soize, Transient dynamics induced by shocks in stochastic structures, in the Proceedings of the Ninth International Conference on Applications of Statistics and Probability in Civil Engineering, ICASP9, Berkeley, San Francisco, July 6-9, 2003: Applications of Statistics an Probability in Civil Engineering, der Kiureghian, Madanat and Pestana (eds), Millpress, Rotterdam, Vol. 1, pp. 267-273, ISBN 9059660048 (2003).

[7] D. Ewins, Modal testing: theory and practice, John Wiley and Sons, Inc., New York, 1984.

[8] C. Fougeaud, A. Fuchs, Statistique, Dunod, Paris, 1967 (2nd ed. 1972).

[9] R. Ghanem, Ingredients for a general purpose stochastic finite elements formulation, Computer Methods in Applied Mechanics and Engineering 168 (1999) 19-34.

[10] R. Ghanem, M. Pellissetti, Adaptive data refinement in the spectral stochastic finite element method, Communications in Numerical Methods in Engineering 18 (2) (2002) 141-151.

[11] R. Ghanem, A. Sarkar, Reduced models for the medium-frequency dynamics of stochastic systems, Journal of the Acoustical Society of America 113 (2) (2003) 834-846.

[12] R. Ghanem, P.D. Spanos, Stochastic Finite Elements: A spectral Approach, Springer-Verlag, New York, 1991.

C. Soize - Computer Methods in Applied Mechanics and Engineering (CMAME) (accepted in March 2004) 
[13] G.H. Golub, C.F. Van Loan, Matrix Computations, Second Edition, The Johns Hopkins University Press, Baltimore, 1989.

[14] I.M. Guelfand, N.Y. Vilenkin, Les Distributions, Tome 4, Applications de l'Analyse Harmonique, Dunod, Paris, 1967.

[15] E.T. Jaynes, Information theory and statistical mechanics, Physical Review 106 (4) (1957) 620-630 \& 108 (2) (1957) 171-190.

[16] J.N. Kapur, H.K. Kesavan, Entropy Optimization Principles with Applications, Academic Press, San Diego, 1992.

[17] M. Kleiber, D.H. Tran, T.D. Hien, The Stochastic Finite Element Method, John Wiley \& Sons, New York, 1992.

[18] K. McConnell, Vibration testing. Theory and practice, Wiley Interscience, New York, 1995.

[19] M.L. Mehta, Random Matrices, Revised and Enlarged Second Edition, Academic Press, New York, 1991.

[20] R. Ohayon, C. Soize, Structural Acoustics and Vibration, Academic press, San Diego, London, 1998.

[21] H.J. Pradlwarter, G.I. Schuëller, G.S. Szekely, Random eigenvalue problems for large systems, Computer and Structures 80 (2002) 2415-2424.

[22] G.I. Schueller (editor), A state-of-the-art report on computational stochastic mechanics, Probabilistic Engineering Mechanics 12(4) (1997) 197-321.

[23] R.J. Serfling, Approximation Theorems of Mathematical Statistics, John Wiley \& Sons, 1980.

[24] C.E. Shannon, A mathematical theory of communication, Bell System Technology Journal 27 (1948) 379-423 \& 623-659.

[25] M. Shinozuka, C.J. Astill, Random eigenvalue problems in structural analysis, AIAA Journal 10 (4) (1972) 456-462.

[26] M. Shinozuka, G. Deodatis, Response variability of stochastic finite element systems, Journal of Engineering Mechanics 114 (3) (1988) 499-519.

[27] C. Soize, A nonparametric model of random uncertainties in linear structural dynamics, Publication du LMA-CNRS, ISBN 2-909669-16-5, 152 (1999)109-138.

[28] C. Soize, A nonparametric model of random uncertainties for reduced matrix models in structural dynamics, Probabilistic Engineering Mechanics 15(3) (2000) 277-294.

[29] C. Soize, Maximum entropy approach for modeling random uncertainties in transient elastodynam-

C. Soize - Computer Methods in Applied Mechanics and Engineering (CMAME) (accepted in March 2004) 
ics, Journal of the Acoustical Society of America 109 (5) (2001) 1979-1996.

[30] C. Soize, Nonlinear dynamical systems with nonparametric model of random uncertainties, Uncertainties in Engineering Mechanics Journal 1 (1) (2001) 1-38, e-journal from Resonance Publication, http://www.resonance-pub.com.

[31] C. Soize, Random matrix theory and non-parametric model of random uncertainties, Journal of Sound and Vibration 263 (2003) 893-916.

[32] C. Soize, Uncertain dynamical systems in the medium-frequency range, Journal of Engineering Mechanics 129 (9) (2003) 1017-1027.

[33] C. Soize, H. Chebli, Random uncertainties model in dynamic substructuring using a nonparametric probabilistic model, Journal of Engineering Mechanics, ASCE 129 (4)(2003) 449-457.

[34] P.D. Spanos, R. Ghanem, Stochastic finite element expansion for random media, Journal of Engineering Mechanics, ASCE 115 (5) (1989) 1035-1053.

[35] G.S. Székely, G.I. Schuëller, Computational procedure for a fast calculation of eigenvectors and eigenvalues of structures with random properties, Computer Methods in Applied Mechanics and Engineering 191 (2001) 799-816.

[36] E. Vanmarcke, M. Grigoriu, Stochastic finite element analysis of simple beams, Journal of Engineering Mechanics, ASCE 109 (5) (1983) 1203-1214.

[37] R.L. Weaver, Spectral statistics in elastodynamics, Journal of the Acoustical Society of America 85 (3) 1989) 1005-1013. 